\title{
La familia Bromeliaceae en
}

\section{México}

\section{Adolfo Espejo-Serna y Ana Rosa López-Ferrari}

Botanical Sciences 96 (3): 533-554, 2018

DOI: $10.17129 /$ botsci.1918

Recibido:

21 de noviembre de 2017

Aceptado:

9 de mayo de 2018

Editor asociado:

Guillermo Ibarra Manriquez

\section{Resumen}

Antecedentes: Las Bromeliaceae mexicanas han despertado el interés de los botánicos desde 1789; su estudio sistemático inició a partir de la década de los setenta del siglo pasado, contando actualmente con avances significativos en su conocimiento taxonómico-florístico.

Pregunta: ¿Cuántas y cuáles especies de Bromeliaceae se presentan en México? ¿Cómo están distribuidas y cuántas son endémicas?

Sitio de estudio: México, 1887-2017.

Métodos: Con base en el estudio de las Bromeliaceae de México, incluyendo recolecciones botánicas, revisión de literatura y consulta, cotejo y determinación de ejemplares en 50 herbarios nacionales y extranjeros, se obtuvieron datos sobre riqueza, endemismo y distribución de sus representantes en el país.

Resultados: En México están representadas cuatro de las ocho subfamilias de Bromeliaceae, 19 géneros, 422 especies y 8 taxones infraespecíficos. Los géneros más ricos en especies son Tillandsia (230/54.5\%), Hechtia (71/16.8 \%) y Pitcairnia (50/11.8\%). Trescientas dieciocho especies, así como los géneros Ursulaea y Viridantha, son endémicos de México; 172 especies son microendémicas. La entidad con más taxones es Oaxaca, seguida por Chiapas, Veracruz y Guerrero y las que cuentan con menor representación son Tlaxcala y Baja California Sur. Baja California, Baja California Sur, Campeche, Ciudad de México, San Luis Potosí, Sonora, Tabasco y Tlaxcala no tienen taxones endémicos estrictos.

Conclusión: Aunque el avance del conocimiento de las Bromeliaceae mexicanas ha sido constante, todavía se requiere de trabajo de exploración y recolección en algunas regiones del país, antes de concluir la flora Bromeliológica Mexicana. También es necesario abordar aspectos de conservación y aprovechamiento sustentable.

Palabras clave: conservación, distribución, endemismo, florística, riqueza de especies.

\section{Abstract:}

Background: Bromeliaceae family in Mexico has been the object of interest by botanists since 1789; their systematic study was approached from the 1970s onwards, and now there are significant advances in its taxonomic-floristic knowledge.

Question: How many and which species of Bromeliaceae occur in Mexico? How they are distributed, and how many are endemic?

Study site: México, 1887-2017.

Methods: Based on the study of the Mexican Bromeliaceae, including botanical collection, literature review, and revision, analysis and determination of specimens in 50 herbaria, data about species richness, Mexican endemics, and distribution of their taxa in the country, were obtained.

Results: In Mexico are represented four of the eight subfamilies of Bromeliaceae, 19 genera, 422 species, and 8 infraespecific taxa. The genera with the highest number of species in the country are Tillandsia (230/54.5\%), Hechtia (71/16.8 \%) and Pitcairnia (50/11.8 \%). 318 of the Bromeliaceae species are endemics to Mexico, as well as Ursulaea and Viridantha genera; 172 species are microendemic. The entity with the highest number of taxa is Oaxaca, followed by Chiapas, Veracruz and Guerrero. Tlaxcala and Baja California Sur have the lowest species number. Baja California, Baja California Sur, Campeche, Ciudad de México, San Luis Potosí, Sonora, Tabasco y Tlaxcala have not strict endemic taxa.

Conclusion: Although progress in the knowledge of Mexican Bromeliaceae has been constant, exploration and recollection work is still required before concluding the Mexican bromeliad flora. It is also necessary to promote studies considering aspects of conservation and sustainable use.

Key words: conservation, distribution, endemism. floristics, species richness.
This is an open access article distributed under the terms of the Creative Commons Attribution License CC BY-NC (4.0) international. 


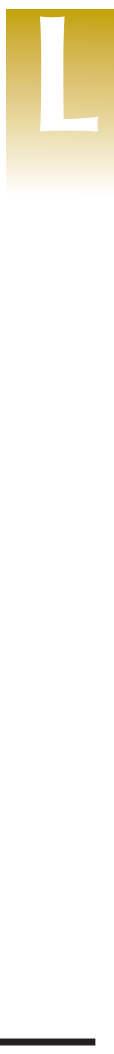

Contribución de los autores:

Adolfo Espejo-Serna concibió la idea original y desarrolló el proyecto; planeó y condujo la recolección de material botánico de Bromeliaceae en varias regiones del país; recolectó e identificó material botánico; revisó, identificó y anotó material herborizado de Bromeliaceae en colecciones institucionales mexicanas y extranjeras; capturó la información en la base de datos; llevó a cabo el análisis de los datos y escribió el manuscrito.

Ana Rosa López-Ferrari colaboró en el desarrollo del proyecto; recolectó e identificó material botánico de la familia procedente de varios estados del país; revisó, identificó y anotó material herborizado de Bromeliaceae en colecciones institucionales mexicanas y extranjeras; capturó la información en la base de datos; Ilevó a cabo el análisis de los datos y escribió el manuscrito. as Bromeliaceae restringen su distribución al continente americano, con excepción de Pitcairnia feliciana (A. Chev.) Harms \& Mildbr., que habita en África. En México, la familia tiene un buen número de representantes epífitos que constituyen elementos característicos de diversos tipos de vegetación, principalmente de los bosques mesófilos de montaña y de los bosques de Quercus. De las especies con hábito terrestre, los representantes de Hechtia habitan preferentemente en matorrales xerófilos y bosques tropicales caducifolios, los de Greigia en bosques mesófilos de montaña, Fosterella micrantha (Lindl.) L.B. Sm. crece en bosques tropicales perennifolios y subcaducifolios y Pitcairnia punicea Scheidw. es una especie estrictamente acuática. Desde el punto de vista económico, algunas especies como la piña (Ananas sativus Schult. \& Schult. f.), el timbiriche o piñuela (Bromelia spp.), la pita (Aechmea magdalenae (André) André ex Baker) y el heno (Tillandsia usneoides (L.) L.), son importantes ya que generan recursos económicos a nivel local y representan una fuente alternativa de ingresos para muchas comunidades rurales, $\mathrm{o}$ incluso algunas de ellas son parte fundamental de la economía estatal (Espejo-Serna \& LópezFerrari 2011).

Los primeros especímenes de la familia Bromeliaceae recolectados en México fueron obtenidos entre 1789 y 1803, por los naturalistas de la Real Expedición Botánica a la Nueva España (Blanco-Fernández-de-Caleya 1987). Desafortunadamente, los resultados de esa magna empresa fueron tardíamente publicados, casi 100 años después (Sessé \& Mociño 1893, 1894), razón por la cual los nombres propuestos por Sessé y Mociño para las especies por ellos recolectadas, son ahora inválidos o sinónimos taxonómicos de especies descritas por otros botánicos como Augustin Pyramus de Candolle o David y George Don. Si bien los nombres que se incluyen en la Flora Mexicana de Sessé \& Mociño (1887) corresponden a 17 especies y dos géneros y los publicados en Plantae Novae Hispaniae (Sessé \& Mociño1893), corresponden a nueve especies y dos géneros, en la revisión reciente del herbario de Sessé y Mociño, conservado en el herbario del Real Jardín Botánico, en Madrid, España (Blanco-Fernández-de-Caleya et al. 2010), encontramos ejemplares de Bromeliaceae, recolectados por los expedicionarios, pertenecientes en total a ocho géneros y 36 especies.

En 1889, Baker, en su Handbook of Bromeliaceae, registró para México 84 especies repartidas en ocho géneros. Mez, en la obra de Casimir de Candolle, Monographiae Phanerogamarum (1897), registró 101 especies en seis géneros. El mismo Mez, en Das Pflanzenreich (Engler 1934-1935), reconoció 143 especies en diez géneros. Smith \& Downs (1974, 1977, 1979), en su importante contribución sobre las Bromeliaceae para la Flora Neotropica, citaron para México 253 especies ubicadas en trece géneros. Esta obra fue, durante los siguientes veinte años, la única y más importante referencia para entender las Bromeliaceae mexicanas.

Como parte de su Sinopsis Florística de las Monocotiledóneas Mexicanas, publicada entre 1994 y 1998, Espejo-Serna \& López-Ferrari (1994), registraron para la República Mexicana 347 especies y 22 géneros. Espejo-Serna et al. (2004) pusieron al día estos datos, registrando entonces 342 especies y 18 géneros para México. En 2012, el autor principal, en su aportación sobre el endemismo en las monocotiledóneas mexicanas, daba cuenta de la existencia de 19 géneros y 400 especies para el país (Figura 1). Por otra parte, como resultado de la aplicación de técnicas de biología molecular (Givnish et al. 1992, 2007, 2011, Barfuss et al. 2005, 2016), se ha modificado la circunscripción infrafamiliar de las Bromeliaceae, pasando de tres a ocho subfamilias, de las cuales tenemos en México representantes de cuatro: Bromelioideae (Aechmea, Androlepis, Billbergia, Bromelia, Greigia, Hohenbergiopsis, Ursulaea y Wittmackia), Pitcairnioideae (Fosterella y Pitcairnia), Hechtioideae (Hechtia) y Tillandsioideae (Catopsis, Guzmania, Pseudalcantarea, Racinaea, Tillandsia, Viridantha, Vriesea y Werauhia).

En los últimos 17 años se han logrado avances significativos en el conocimiento taxonómico-florístico de la familia, describiéndose para el país, entre 2000 y 2017, 78 especies (18.4\% del total conocido), es decir un promedio de 5 por año (Ehlers 2001, 2002a, b, c, 2003a, b, c, 2005a, b, c, 2006a, b, c, 2009, 2011, Espejo-Serna 2002, Ramírez-Morillo \& Carnevali 2003, 2007a, b, Espejo-Serna \& López-Ferrari 2005, 2007, 2008, 2009, 2010, 2015, López-Ferrari et al. 2006, 2008, 2011, Krömer et al. 2007, Espejo-Serna et al. 2007a, b, 2011, Espejo et al. 2008, Flores-Cruz \& Diego-Escobar 2008, Ramírez-Morillo 2008, López-Ferrari \& Espejo-Serna 2009, 2013, 2014b, Martínez-Correa et al. 2010, 2014, Burt-Utley et al. 2011, Pinzón et al. 2011, Ramírez-Morillo et al. 2011, 2013, 2014, 2015, Ramírez-Morillo \& Jiménez Nah, 2012, 
Figura 1. Registros contenidos, hasta el momento, en la base de datos de Bromeliaceae mexicanas.

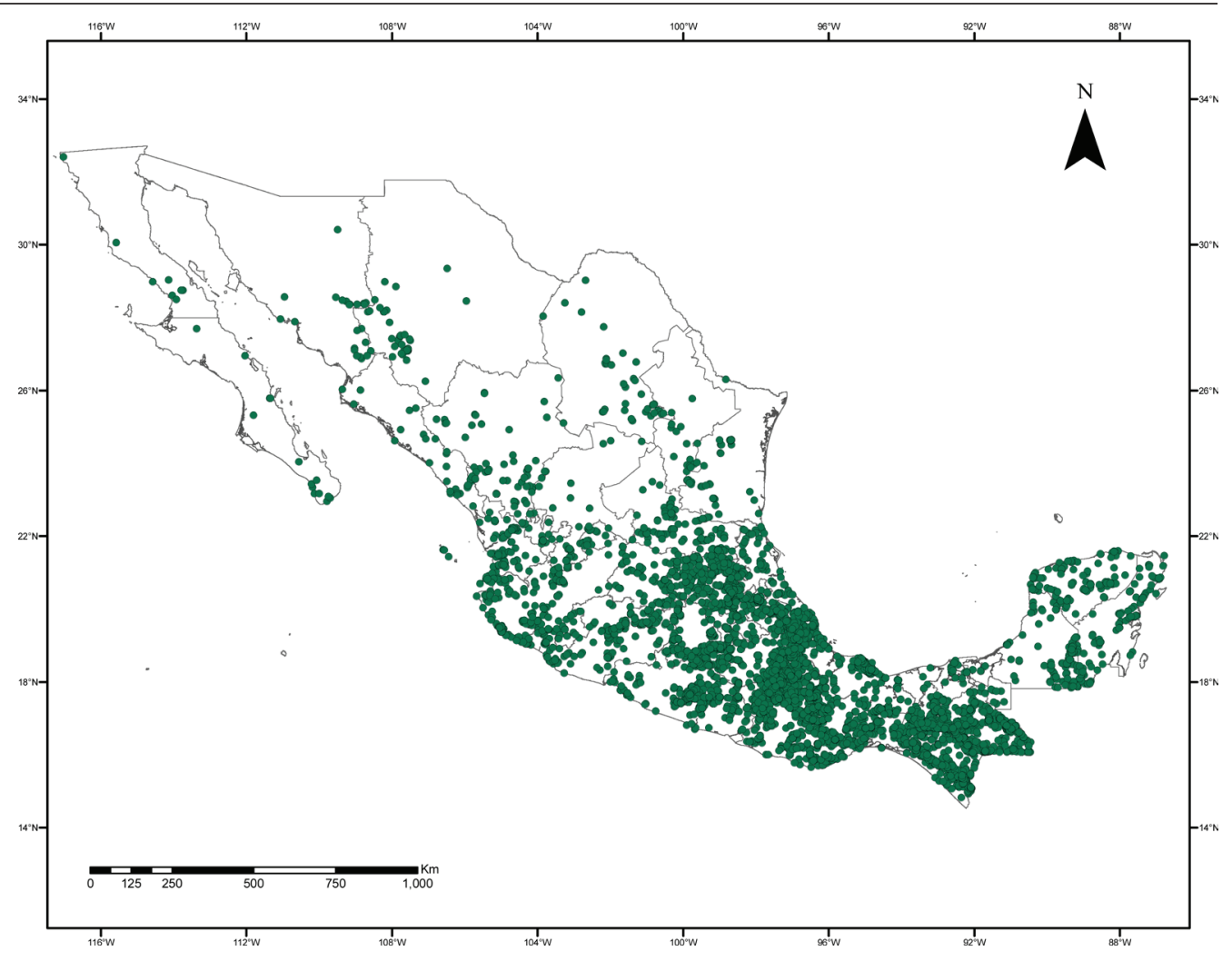

Burt-Utley 2012, García-Ruiz et al. 2014, Hernández-Cárdenas et al. 2014, González-Rocha et al. 2014, 2015, García-Martínez \& Beutelspacher 2017). Asimismo, la exploración botánica en diversas regiones del territorio nacional, la recolección sistemática de ejemplares en el marco de diversos proyectos florísticos y el estudio de ejemplares herborizados, han permitido que en este momento el conocimiento sobre la familia sea bastante mejor que hace algunos años, sin que esto signifique que está completo.

Actualmente, existen diversos tratamientos florísticos de la familia para algunos estados o regiones del país: North American Flora (Smith 1938), Flora Neotropica (Smith \& Downs 1974, 1977, 1979), Flora Novogaliciana (McVaugh 1989), Flora Fanerogámica del Valle de México (Victoria-Hernández 2001, Espejo-Serna \& López-Ferrari 2004), Flora Mesoamericana (Utley \& Burt-Utley 1994), Bromeliaceae de Guerrero (Pulido-Esparza et al. 2004), Flora de la porción mexicana de la Península de Yucatán (Ramírez-Morillo et al. 2004), Flora de Veracruz (Espejo-Serna et al. 2005), Bromeliad flora of Oaxaca (Espejo-Serna et al. 2007c), Flora del Bajío y de Regiones Adyacentes (Espejo-Serna et al. 2010), Flora del Valle de Tehuacán-Cuicatlán (López-Ferrari \& Espejo-Serna 2014a), Las Bromeliaceae del estado de Morelos (GonzálezRocha et al. 2016) y Bromeliad Flora of Chiapas State, Mexico: Richness and Distribution (Espejo-Serna et al. 2017).

Existen también numerosas contribuciones florísticas que, sin tener como grupo principal de estudio a las Bromeliaceae, aportan datos sobre la distribución de sus especies (e.g., Alanís-Méndez et al. 2007, García-Franco \& Toledo-Aceves 2008, García-Franco et al. 2008). Igualmente, existen numerosas tesis, de licenciatura o posgrado, sobre aspectos florísticos, taxonómicos, morfológico-anatómicos o etnobotánicos (e.g., Villa-Guzmán 2007, Cach-Pérez 2008, Viccon-Esquivel 2009, Salazar-Rodríguez 2010). En lo relativo al conocimiento filogenético de las Bromeliaceae mexicanas, hasta el momento existen pocas contribuciones publicadas por botánicos nacionales (Escobedo-Sarti et al. 2013, Pinzón et al. 2016) y hay otros especialistas trabajando estos aspectos, tanto en la Universidad Autónoma Metropolitana Iztapalapa $(\mathrm{Ca}$ topsis, Pitcairnia, Tillandsia y Viridantha), como en el Instituto de Biología de la Universidad Nacional Autónoma de México (Tillandsia) y en el Centro de Investigación Científica de Yu- 
catán (Hechtia). Existen también diversas contribuciones de taxónomos extranjeros (Barfuss et al 2005, Givnish et al. 2011, 2014, Givnish 2017, Schütz et al. 2016) y más recientemente, Barfuss et al. (2016) publicaron una revisión taxonómica sobre la subfamilia Tillandsioideae, utilizando caracteres morfológicos y moleculares, que ha conducido a algunos cambios en la circunscripción genérica de algunos de sus integrantes, así como la propuesta de nuevas tribus, subtribus, subgéneros y 10 géneros nuevos segregados de Mezobromelia, Tillandsia y Vriesea, ninguno de ellos presente en México, con excepción de Pseudalcantarea, cuyas tres especies crecen en el país.

Por lo que se refiere a los botánicos que han abordado el estudio de esta familia, indudablemente uno de los taxónomos que contribuyó de manera sustancial al conocimiento de las Bromeliaceae de México fue Lyman B. Smith (1904-1997) (e.g., Smith 1938, Smith \& Downs 1974, 1977, 1979), quien por muchos años fue el único especialista dedicado a este grupo botánico. También es importante mencionar a Renate Ehlers, de nacionalidad alemana, quién ha descrito muchas especies endémicas habitantes de riscos y cantiles (e.g., Ehlers 2001, 2002a, b, c, 2003a, b, c, 2005a, b, c, 2006a, b, c, 2009, 2011), a Adolfo Espejo Serna y colaboradores de la Universidad Autónoma Metropolitana Iztapalapa (e.g., Espejo-Serna 2002, 2012, Espejo-Serna \& López-Ferrari 1994, 1998, 2004, 2005, 2007, 2009, 2010, 2015, Espejo et al 2008, EspejoSerna et al. 2004, 2005, 2007a, b, c, 2010, 2011, González-Rocha et al. 2016, López-Ferrari \& Espejo-Serna 2009, 2013, 2014a, b, López-Ferrari et al. 2006, 2008, 2009, 2011, Martínez-Correa et al. 2010, 2014, Ramírez-Morillo et al. 2011, Hernández-Cárdenas et al. 2014, Krömer et al. 2007), a Eizi Matuda (1894-1978), japonés nacionalizado mexicano e investigador del Instituto de Biología de la Universidad Nacional Autónoma de México, reconocido por sus aportaciones al conocimiento de las monocotiledóneas mexicanas (e.g., Matuda 1952, 1957, 1973, 1975, 1977), a Ivón Ramírez Morillo y colaboradores, del Centro de Investigación Científica de Yucatán (Ramírez-Morillo 2008, Ramírez-Morillo et al. 2003, 2004, 2007a, b, 2012, 2013, 2014, 2015) y a John Utley y Kahtleen Burt-Utley, de la Universidad de Nueva Orleans quienes han contribuido sustancialmente al conocimiento del género Hechtia (e.g., Burt-Utley 2012, Burt-Utley et al. 2011, Utley \& Burt-Utley 1994).

Entre otros taxónomos nacionales estudiosos de las bromeliáceas mexicanas están Carlos Rommel Beutelspacher (e.g., Beutelspacher. 1971, 1989, Beutelspacher \& Farrera 2007, Beutelspacher \& Moreno Molina 2011, Beutelspacher \& López-Velázquez 2008), de la Universidad de Ciencias y Artes del Estado de Chiapas, quien ha descrito algunas novedades para la familia y abordado además cuestiones sobre los usos de las bromelias por las etnias chiapanecas; Nancy Martínez Correa (Martínez-Correa et al. 2010, 2014), Edith González Rocha (González-Rocha et al. 2014, 2015, 2016) y Rodrigo Alejando Hernández Cárdenas (Hernández-Cárdenas et al. 2014), de la Universidad Autónoma Metropolitana Iztapalapa, quienes han trabajado, en colaboración con Adolfo Espejo, en estudios florísticos estatales y en la revisión de varios géneros de la familia en México, como Catopsis, Hechtia, Pitcairnia y Viridantha; Claudia Hornung Leoni (e.g., Hornung-Leoni et al. 2011) de la Universidad Autónoma del Estado de Hidalgo, que está dedicada al estudio de la Bromelioflora hidalguense; Juan P. Pinzón (Pinzón et al. 2011, 2012), de la Universidad Autónoma de Yucatán, quien abordó el estudio taxonómico-filogenético del complejo de Tillandsia utriculata y Carolina Granados Mendoza (Granados-Mendoza et al. 2016), del Instituto de Biología de la Universidad Nacional Autónoma de México, quien realizó su tesis doctoral con el estudio taxonómico-filogenético del grupo de Tillandsia erubescens y quien continua estudiando la sistemática del género (Granados-Mendoza et al. 2017).

Entre los botánicos extranjeros se puede hacer mención de Walter Till (Till \& Schatzl 1981, Till 1992, Till et al.1989) del Instituto de Botánica de la Universidad de Viena, de Bruce K. Holst (Espejo-Serna et al. 2004), de Marie Selby Botanical Gardens y de Sue Sill, actualmente dedicada a la pintura artística y de naturaleza, cuyo trabajo sobre la clasificación de Tillandsia subgénero Tillandsia en México (Gardner 1982, 1986), es fundamental para el entendimiento del mismo.

El estudio de las Bromeliaceae de México ha sido abordado también por estudiosos de otras disciplinas como la Ecología y la Etnobotánica. Entre los colegas que están participando en dichos estudios se encuentran Thorsten Krömer (Krömer et al. 2008), del Centro de Investigaciones Tropicales de la Universidad Veracruzana, Alejandro Flores Palacios (Flores-Palacios et al. 
2011), del Centro de Investigación en Biodiversidad de la Universidad Autónoma de Morelos, José Guadalupe García Franco (García-Franco \& Toledo-Aceves 2008, García-Franco et al. 2008) del Instituto de Ecología, A. C., Xalapa y Demetria Mondragón Chaparro (Mondragón \& Villa-Guzmán 2008, Mondragón-Chaparro \& Ticktin 2011), del Centro Interdisciplinario de Investigación para el Desarrollo Integral Regional Unidad Oaxaca. Con base en lo anterior, el objetivo de la presente contribución es actualizar el conocimiento florístico de Bromeliaceae en México para concer cuántas y cuáles especies de la familia se presentan en el país, documentar su distribución en los distintos estados que integran el territorio nacional y cuantificar el número de especies endémicas mexicanas.

\section{Materiales y métodos}

A lo largo de más de 25 años de estudio sobre la familia hemos recabado la información que respalda los datos presentados en esta contribución. El trabajo ha incluido la realización de viajes de recolección a diversas localidades del país, considerando la visita a muchas de las localidades tipo, la revisión de toda la literatura relativa a aspectos taxonómicos, florísticos y nomenclaturales de las Bromeliaceae de México, así como estancias de trabajo en diversas instituciones botánicas, con el propósito de revisar, cotejar y determinar material herborizado de bromelias mexicanas. En total, hemos consultado material en cerca de 50 colecciones institucionales nacionales y extranjeras: BM, BR, CAS, CH, CHAP, CHAPA, CHIP, CIB, CICY, CIIDIR, CIQRO, CODAGEM, CORU, ENCB, F, FCME, GH, HAL, HEID, HEM, HUMO, IBUG, IEB, IZTA, K, LE, LL, MA, MANCH, MEXU, MICH, MO, NY, OAX, P, QMEX, SEL, SERO, TEX, UAMIZ, UC, US, USON, USF, WU, XAL (Thiers [actualizado continuamente]), incluyendo material Tipo y hemos elaborado una base de datos de nombres y de especímenes. Cabe mencionar que, en todos los ejemplares revisados en los herbarios mencionados previamente, ha quedado debidamente indicada esta actividad. Con base en la información geográfica recabada de cerca de 31,500 ejemplares herborizados, hemos realizado análisis de la distribución conocida, a nivel municipal, estatal, de áreas naturales protegidas y de cuadrículas de $1 \times$ $1^{\circ}$ de todas las especies de la familia presentes en el país. Los datos de ubicación geográfica de estos especímenes han sido corroborados utilizando mapas topográficos 1:50 000 (INEGI 2016) y los programas Google Earth (Google 2016) y Arc View v. 9.3.1 (ESRI 2009).

\section{Resultados}

Diversidad y endemismo. En México, la familia Bromeliaceae incluye 19 géneros, 422 especies y 8 taxones infraespecíficos, lo cual arroja la cifra de 430 taxa (Apéndice 1), así como 960 nombres y 538 sinónimos tanto homotípicos como heterotípicos. Los géneros con mayor número de especies son: Tillandsia (230 spp./54.5\% del total de especies de la familia en el país) Hechtia (71/16.8 \%) y Pitcairnia (50/11.8 \%). Androlepis, Hohenbergiopsis y Wittmackia están representados en México únicamente por un taxón. De las 422 especies mexicanas, 318 (75.3\%), son endémicas de México, en tanto que dos géneros presentan el mismo estatus de endemismo: Ursulaea (2 spp.) y Viridantha (12). Además, las tres especies de Greigia presentes en México son endémicas al país, en tanto que $98.6 \%$ de las especies de Hechtia, $80 \%$ de las de Pitcairnia y $76.9 \%$ de las de Tillandsia también restringen su distribución al territorio nacional. En lo que se refiere a los endemismos estrictos por estado, Oaxaca ocupa el primer lugar con 56, seguido por Chiapas (24) y Guerrero (21). Baja California, Baja California Sur, Campeche, Ciudad de México, San Luis Potosí, Sonora, Tabasco y Tlaxcala no cuentan con taxones endémicos estrictos. El género Hechtia es el único de la subfamilia Hechtioideae y ambos son endémicos de Megaméxico 3 (Rzedowski 1991).

Distribución geográfica. La entidad con mayor número de especies es Oaxaca, con 197, seguida por Chiapas (136), Veracruz (98) y Guerrero (89), en tanto que Tlaxcala (5) y Baja California Sur (3) cuentan con el menor número de especies registradas y en Baja California sólo se presenta Tillandsia recurvata. En la Tabla 1 se presenta la información relativa al número de especies de Bromeliaceae registradas en cada entidad, así como el porcentaje que éstas representan con 
Tabla 1. Número total de especies de Bromeliaceae y número de endémicas estatales (microendémicas) registradas en cada estado.

\begin{tabular}{|c|c|c|}
\hline Estado & N. ${ }^{\circ}$ especies & N. ${ }^{\circ}$ endémicas \\
\hline Oaxaca & $197(46.6 \%)$ & $56(28.5 \%)$ \\
\hline Chiapas & $135(31.9 \%)$ & $24(17.7 \%)$ \\
\hline Veracruz & $96(22.7 \%)$ & $10(10.4 \%)$ \\
\hline Guerrero & $90(21.3 \%)$ & $21(23.3 \%)$ \\
\hline Jalisco & $78(18.4 \%)$ & $12(15.3 \%)$ \\
\hline Puebla & $67(15.8 \%)$ & $7(10.4 \%)$ \\
\hline Michoacán & $59(13.9 \%)$ & $6(10.1 \%)$ \\
\hline Estado de México & $45(10.6 \%)$ & $5(11.1 \%)$ \\
\hline Nayarit & $41(9.7 \%)$ & $1(2.4 \%)$ \\
\hline Morelos & $36(8.5 \%)$ & $4(11.1 \%)$ \\
\hline Colima & $35(8.2 \%)$ & $2(5.7 \%)$ \\
\hline Hidalgo & $35(8.2 \%)$ & $4(11.4 \%)$ \\
\hline Querétaro & $33(7.8 \%)$ & $1(3.0 \%)$ \\
\hline Guanajuato & $30(7.1 \%)$ & $1(3.3 \%)$ \\
\hline Quintana Roo & $29(6.8 \%)$ & $3(10.3 \%)$ \\
\hline Tabasco & $29(6.8 \%)$ & 0 \\
\hline San Luis Potosí & $27(6.3 \%)$ & 0 \\
\hline Campeche & $25(5.9 \%)$ & 0 \\
\hline Tamaulipas & $24(5.6 \%)$ & $2(8.3 \%)$ \\
\hline Sinaloa & $21(4.9 \%)$ & $3(14.2 \%)$ \\
\hline Durango & $18(4.2 \%)$ & $3(16.6 \%)$ \\
\hline Zacatecas & $18(4.2 \%)$ & $3(16.6 \%)$ \\
\hline Yucatán & $16(3.7 \%)$ & $1(6.2 \%)$ \\
\hline Sonora & $11(2.6 \%)$ & 0 \\
\hline Nuevo León & $10(2.3 \%)$ & $2(20 \%)$ \\
\hline Aguascalientes & $10(2.3 \%)$ & $1(10 \%)$ \\
\hline Chihuahua & $8(1.8 \%)$ & $2(25 \%)$ \\
\hline Ciudad de México & $8(1.8 \%)$ & 0 \\
\hline Coahuila & $8(1.8 \%)$ & $1(12.5 \%)$ \\
\hline Tlaxcala & $5(1.1 \%)$ & 0 \\
\hline Baja California Sur & $3(0.7 \%)$ & 0 \\
\hline Baja California & $1(0.2 \%)$ & 0 \\
\hline
\end{tabular}

respecto al total de taxones de la familia presentes en México. De igual manera se incluye en la Tabla 1 el número de especies endémicas por estado (microendémicas), junto con el porcentaje que éstas representan con respecto a las registradas en la entidad correspondiente.

\section{Discusión}

La familia Bromeliaceae agrupa a nivel mundial 76 géneros y 3,547 especies (Gouda et al. continuamente actualizado), de las cuales en México tenemos representados 19 y 422, respectivamente, lo que corresponde a $25 \%$ del total genérico y a $11.8 \%$ del total específico. A nivel nacional, las Bromeliaceae ocupan el undécimo lugar en riqueza específica entre las angiospermas y el tercer sitio entre las monocotiledóneas, después de Orchidaceae y Poaceae (Espejo-Serna 2012, Villaseñor 2016). En lo relativo a la representación genérica de la familia en nuestro país, 
Tillandsia ocupa el primer sitio con 230 especies, lo que lo ubica como el tercer género más diverso a nivel nacional, después de Salvia y Euphorbia (Villaseñor 2016). Los otros géneros de Bromeliaceae con más especies presentes en México son Hechtia con 71 y Pitcairnia con 50.

Por lo que respecta al endemismo, 318 de las 422 especies de Bromeliaceae mexicanas restringen su distribución al territorio nacional, lo que corresponde a $75.3 \%$, cifra que rebasa la estimada por Villaseñor (2016) para las plantas vasculares mexicanas en su conjunto (49.8\%). Además, 173 especies son microendémicas, es decir, se conocen únicamente de un estado, ocupando los primeros lugares en este rubro Oaxaca, Chiapas y Guerrero (Gomez-Escamilla et al. en preparación). Lo arriba expuesto refuerza la necesidad de generar planes de manejo y/o conservación de dichas especies, así como de llevar a cabo estudios específicos sobre sus requeriemientos ecológicos y biología reproductiva.

Si bien el avance en el conocimiento florístico de esta importante familia en México ha sido constante en años recientes, existen todavía algunos grupos que requieren de la atención de los taxónomos, en particular en los géneros Pitcairnia y Tillandsia, en los cuales se presentan complejos de especies de difícil delimitación y reconocimiento, sobre todo cuando se trabaja con material herborizado. En estos casos, es necesario realizar estudios de campo para trabajar con plantas vivas, lo que facilitará el análisis de características no disponibles en los ejemplares secos y prensados y permitirá además observar las variaciones a nivel poblacional.

Tambien es necesario explorar varias regiones del país que requieren aún de trabajo de recolección. En la Figura 1 se incluyen todos los registros de bromeliáceas incorporados hasta el momento en la base de datos del proyecto. Como puede apreciarse, existen áreas que todavía requieren de un importante esfuerzo de recolección, entre las que podemos mencionar a algunas regiones de los estados del norte del país como Durango, Sinaloa y Sonora, las porciones de tierra caliente de los estados de Guerrero (Pulido-Esparza et al. 2004) y Michoacán, algunas zonas poco recolectadas de Oaxaca (García-Mendoza 2004, Espejo-Serna et al. 2007c) y Jalisco, así como algunas áreas del noroeste y de la Sierra Madre de Chiapas (Espejo-Serna et al. 2017). De igual manera, es necesario estudiar la flora de riscos, peñascos, acantilados y cañones, ya que son sitios que, por su difícil acceso, reciben poca atención de los recolectores y constituyen hábitats preferidos por muchos de los representantes de la familia. De hecho una buena parte de las novedades mexicanas recientes de Bromeliaceae proceden de este tipo de lugares (EspejoSerna et al. 2011, Flores-Argüelles et al. 2017).

Aunque se piensa que las Bromeliaceae en su conjunto no están expuestas todavía a las amenazas antrópicas que enfrentan las orquídeas, existen algunas regiones del país, particularmente en los estados de Chiapas, Oaxaca y Veracruz (Beutelspacher 1989, Beutelspacher \& FarreraSarmiento 2007, Flores-Palacios \& Valencia-Díaz 2007), en las que las poblaciones silvestres de varias especies, particularmente de los géneros Tillandsia y Catopsis, han sido mermadas sistemáticamente ya que son utilizadas indiscriminadamente por las comunidades humanas que habitan en las áreas en las que se distribuyen. De aquí que sea impostergable impulsar el estudio de la familia abordando aspectos de conservación y aprovechamiento sustentable (Wolf \& Konings 2001).

También es importante impulsar los estudios biogeográficos de las Bromeliaceae mexicanas. Recientemente hemos abordado, a través del proyecto Distribución Potencial de las Especies de Bromeliaceae Mexicanas (Espejo Serna 2017), apoyado por la CONABIO, aspectos de modelado, generando mapas de distribución potencial (GARP y MAXENT) para todos los taxa de la familia endémicos de México. A manera de ejemplo, se incluyen en la Figura 2, los datos obtenidos para Viridantha atroviridipetala (Matuda) Espejo. Los modelos de distribución potencial (MDP) relacionan la distribución geográfica conocida (latitud/longitud) de las especies con diversas variables ecológico-climáticas, para identificar áreas en las cuales las especies podrían potencialmente prosperar, asumiendo que la distribución conocida de un taxon proporciona información suficiente sobre sus requerimientos ecológicos (Kadmon et al. 2003). Las áreas predichas pueden ser visualizadas y proyectadas en un espacio geográfico con la ayuda de sistemas de información geográfica (SIG's) (Araújo \& Guisan 2006, Kearney 2006). Dichos modelos han demostrado su utilidad en estudios que evalúan patrones de distribución de organismos, como son algunos análisis biogeográficos, ecológicos o de conservación (Anderson et al. 2003). 
Figura 2. Distribución conocida (puntos) y potencial (color verde) de Viridantha atroviridipetala (Matuda) Espejo.

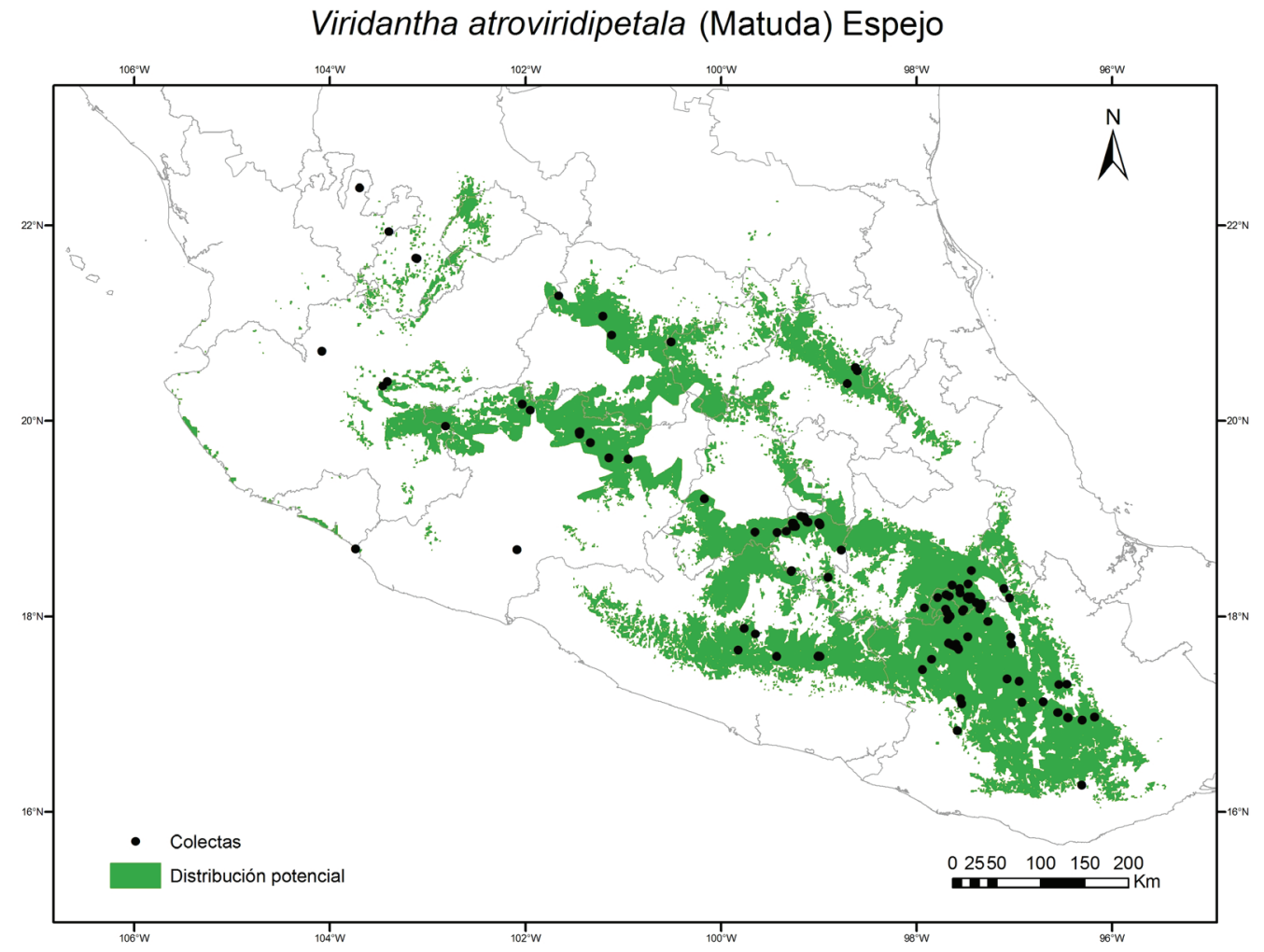

Finalmente, queremos mencionar que, si bien hay botánicos jóvenes que se están formando en el campo de la taxonomía y la sistemática de Bromeliaceae, éstos deben enfrentarse, como lo hacemos a diario muchos taxónomos activos, a diversos problemas tales como la falta de fuentes de financiamiento suficientes para este tipo de proyectos y la incomprensión, por parte de las autoridades institucionales y de los pares académicos, sobre la importancia que tienen los estudios taxonómicos que generan conocimiento acerca del capital natural de México, entendido éste como bien lo ha señalado Sarukhán (2008), no sólo como la biodiversidad representada por especies y comunidades, sino también como los servicios que los ecosistemas proveen a la sociedad en sus distintos aspectos.

\section{Agradecimientos}

Deseamos expresar nuestro agradecimiento a Guillermo Ibarra Manríquez, editor de sección, y a Abisaí García Mendoza e Hilda Flores Olvera por la revisión, los comentarios y las observaciones al trabajo, el cual fue presentado en parte en el Simposio Familias diversas de la Flora de México, realizado en el marco del XX Congreso Mexicano de Botánica. De igual manera y aunque lo hemos hecho en reiteradas ocasiones, deseamos agradecer a todos los encargados (pasados y actuales) de los herbarios revisados, por todas las facilidades que siempre nos otorgaron para la consulta irrestricta del material de Bromeliaceae depositado en las colecciones a su cargo. Finalmente, agradecemos a Ivonne Nayeli Gomez Escamilla quién participó activamente en la realización de los mapas que acompañan al texto.

\section{Literatura citada}

Alanís-Méndez JL, Muñoz-Arteaga FO, López-Ortega M, Cuervo-López L, Raya-Cruz BE. 2007. Aportes al conocimiento de las epífitas (Bromeliaceae, Cactaceae y Orchidaceae) en dos tipos de vegetación del municipio de Pánuco, Veracruz, México). Revista UDO Agrícola 7: 160-174.

Anderson RP, Lew D, Peterson AT. 2003. Evaluating predictive models of species' distributions: criteria for selecting optimal models. Ecological Modelling 162: 211. DOI: 10.1016/S0304-3800(02)00349-6 
Araújo MB, Guisan A. 2006. Five (or so) challenges for species distribution modelling. Journal of Biogeography 33: 1677-1688. DOI: 10.1111/j.1365-2699.2006.01584.x

Baker JG. 1889. Handbook of the Bromeliaceae. London: George Bell \& Sons.

Barfuss MH, Samuel R, Till W, Stuessy TF. 2005. Phylogenetic relationships in subfamily Tillandsioideae (Bromeliaceae) based on DNA sequence data from seven plastid regions. American Journal of Botany 92: 337-351. DOI: 10.3732/ajb.92.2.337

Barfuss MHJ, Till W, Leme EMC, Pinzón JP, Manzanares JM, Halbritter H, Samuel R, Brown GK. 2016. Taxonomic revisión of Bromeliaceae subf. Tillandsioideae based on a multi-locus DNA sequence phylogeny and morphology. Phytotaxa 279: 1-97. DOI: 10.11646/phytotaxa.279.1

Beutelspacher CR. 1971. Aechmea bracteata (Sw.) Griseb. var. pacifica Beutelspacher, var. nov. (Bromeliaceae). Cactáceas y Suculentas Mexicanas 16: 44-46.

Beutelspacher CR. 1989. Bromeliáceas asociadas a la fiesta del "niño florero" en Chiapa de Corzo, Chiapas. Cactáceas y Suculentas Mexicanas 34: 44-47.

Beutelspacher BCR, Farrera-Sarmiento O. 2007. Tradición vs. Conservación: La "Topada de la Flor". Lacandonia 1: 109-115.

Beutelspacher BCR, López-Velázquez G. 2008. Una nueva especie de Pitcairnia L'Hér. de Chiapas, México (Bromeliaceae: Pitcairnioideae). Lacandonia 2: 29-31.

Beutelspacher BCR, Moreno-Molina I. 2011. Orquidáceas y Bromeliáceas del Parque Nacional Lagunas de Montebello, Chiapas, México. Lacandonia 5: 87-102.

Blanco-Fernández-de-Caleya P. 1987. Los herbarios de Sessé y Mociño. In: Sánchez B, Puig-Samper MA, de la Sota J. eds. La Real Expedición Botánica a Nueva España 1787-1803. Madrid: Real Jardín Botánico. 253-260.

Blanco-Fernández-de-Caleya P, Espejo-Serna A, López-Ferrari AR. 2010. Catálogo del Herbario de la Real Expedición Botánica de Nueva España (1787-1803) conservado el Real Jardín Botánico de Madrid. Madrid: Consejo Superior de Investigaciones Científicas. Real Jardín Botánico. ISBN: 978-8400-09262-7

Burt-Utley K. 2012. Contributions toward a revision of Hechtia (Bromeliaceae, Pitcairnioideae) II. New and noteworthy Hechtia species from Oaxaca, Mexico. Phytoneuron 69: 1-14.

Burt-Utley K, Utley JF, García-Mendoza A. 2011. Contributions toward a revision of Hechtia (Bromeliaceae, Pitcairnioideae). I. New and noteworthy species of Hechtia from Mexico. Phytoneuron 59: 1-17.

Cach-Pérez MJ. 2008. Influencia ambiental sobre la fisiología y anatomía foliar de Tillandsia heterophylla, bromelia endémica de México. Msc. Thesis. Instituto de Ecología, A.C.

Ehlers R. 2001. Tillandsia atenangoensis Ehlers \& Wuelfinghoff. Bromelie 2: 45-48.

Ehlers R. 2002a. Tillandsia zoquensis Ehlers spec. nov. Bromelie 1: 18-21.

Ehlers R. 2002b. Tillandsia crista-gallii Ehlers. Bromelie 2: 32-36.

Ehlers R. 2002c. Tillandsia loxichaensis Ehlers spec. nov. Bromelie 3: 78-82.

Ehlers R. 2003a. A new Tillandsia from Oaxaca, Mexico. Journal of the Bromeliad Society 53: 16-20.

Ehlers R. 2003b. Tillandsia loma-blancae Ehlers et Lautner. Bromelie 1: 21-24.

Ehlers R. 2003c. Tillandsia polita LB. Smith var. elongata Ehlers var. nov. Bromelie 2: 45-48.

Ehlers R. 2005a. Tillandsia lagunaensis Ehlers, spec. nov. Bromelie 3: 80-84.

Ehlers R. 2005b. Tillandsia zacualpanensis Ehlers \& Wülfinghoff spec. nov. Bromelie 1: 12-16.

Ehlers R. 2005c. Tillandsia zaragozaensis R. Ehlers spec. nov. Bromelie 2: 44-48.

Ehlers R. 2006a. Tillandsia huamelulaensis: a new species from the State of Oaxaca, Mexico. Journal of the Bromeliad Society 56: 56-59.

Ehlers R. 2006b. Tillandsia nicolasensis: a new species from coastal Jalisco, Mexico. Journal of the Bromeliad Society 56: 70-72.

Ehlers R. 2006c. Tillandsia pseudooaxacana Ehlers spec. nov. Die Bromelie 2006: 92-95.

Ehlers, R. 2009. The green-blooming, small, grey Tillandsias from Mexico. Die Bromelie Sonderheft 6:1-144.

Ehlers R. 2011. Tillandsia chapalillaensis Ehlers \& J. Lautner spec. nov. Journal of the Bromeliad Society 61: 169-173.

Escobedo-Sarti J, Ramírez I, Leopardi C, Carnevali G, Magallón S, Duno R, Mondragón D. 2013. A phylogeny of Bromeliaceae (Poales, Monocotyledoneae) derived from an evaluation of nine supertree methods. Journal os Systematic and Evolution 51: 743-757. DOI: 10.1111/jse.12044

Espejo A, López-Ferrari AR, Till W. 2008. Dos nuevas especies de Tillandsia (Bromeliaceae) de México. Acta Botanica Mexicana 85: 45-62. DOI: 10.21829/abm85.2008.1072

Espejo-Serna A. 2002. Viridantha, un género nuevo de Bromeliaceae (Tillandsioideae) endémico de México. Acta Botanica Mexicana 60: 25-35. DOI: 10.21829/abm60.2002.901

Espejo-Serna A. 2012. El endemismo en las Liliopsida Mexicanas. Acta Botanica Mexicana 100: 195-257. DOI: $10.21829 / \mathrm{abm} 100.2012 .36$ 
Espejo-Serna A. 2017. Distribución potencial de las especies de Bromeliaceae mexicanas. Universidad Autónoma Metropolitana-Iztapalapa. Informe final. SNIB-CONABIO, proyecto No. JM008. Ciudad de México

Espejo-Serna A, López-Ferrari AR. 1994. Las monocotiledóneas mexicanas, una sinopsis florística 1. Lista de referencia, parte III. Bromeliaceae, Burmanniaceae, Calochortaceae y Cannaceae. Ciudad de México: Consejo Nacional de la Flora de México, Universidad Autónoma Metropolitana y Comisión Nacional para el Conocimiento y Uso de la Biodiversidad. México. ISBN: 9686144102

Espejo Serna A, López-Ferrari AR. 1998. Current floristic and phytogeographic knowledge of Mexican Bromeliaceae. Revista de Biología Tropical 46: 493-513.

Espejo Serna A, López-Ferrari AR. 2004. Notas sobre la familia Bromeliaceae en el Valle de México. Acta Botanica Mexicana 67: 49-57. DOI: 10.21829/abm67.2004.973

Espejo Serna A, López-Ferrari AR. 2005. Una nueva especie de Tillandsia (Bromeliaceae) del occidente de México. Acta Botanica Mexicana 72: 53-64. DOI: 10.21829/abm72.2005.1001

Espejo Serna A, López-Ferrari AR. 2007. Una nueva especie de Tillandsia (Bromeliaceae) de la Sierra del Halo, Tecalitlán, Jalisco, México. Acta Botanica Mexicana 80: 41-49. DOI: 10.21829/ abm80.2007.1045

Espejo-Serna A, López-Ferrari AR. 2009. Tillandsia magnispica (Bromeliaceae: Tillandsioideae) a new species from Oaxaca, Mexico. Acta Botanica Mexicana 86: 1-7. DOI: 10.21829/abm86.2009.1075

Espejo-Serna A, López-Ferrari, AR. 2010. Pitcirnaia yocupitziae (Bromeliaceae; Pitcairnioideae), notable nueva especie de Guerrero, México. Acta Botanica Mexicana 93: 103-110. DOI: 10.21829/ abm93.2010.276

Espejo-Serna A, López-Ferrari AR. 2011. Diversidad y distribución de las Bromeliaceae. In: CONABIO [Comisión Nacional para el Conocimiento y Uso de la Biodiversidad]. La Biodiversidad en Veracruz. Estudio de Caso. Volumen II Diversidad de Especies: Conocimiento Actual. México: CONABIO, Gobierno del Estado de Veracruz, Universidad Veracruzana, Instituto de Ecología, A.C. 177-189. ISBN: 978-607-7607-51-9

Espejo-Serna A, López-Ferrari AR. 2015. Pitcairnia queroana (Pitcairnioideae, Bromeliaceae), a striking new species from Chiapas state, Mexico. Phytotaxa 230: 287-292. DOI: 10.11646/phytotaxa.230.3.8

Espejo-Serna A, López-Ferrari AR, Ceja Romero J, Mendoza-Ruiz A. 2011. Una nueva especie de $T i$ llandsia (Bromeliaceae) del cañón de Escahuasco, Puebla, México. Acta Botanica Mexicana 96: 73-77. DOI: $10.21829 / \mathrm{abm} 96.2011 .260$

Espejo-Serna A, López-Ferrari AR, Martínez-Correa N, Pulido-Esparza VA. 2007c. Bromeliad Flora of Oaxaca, Mexico: Richness and Distribution. Acta Botanica Mexicana 81: 71-147. DOI: 10.21829/ abm81.2007.1052

Espejo-Serna A, López-Ferrari AR, Martínez-Correa N, Pulido-Esparza VA. 2017. Bromeliad Flora of Chiapas State, Mexico: Richness and Distribution. Phytotaxa 310: 1-74. DOI: 10.11646/phytotaxa.310.1.1

Espejo-Serna A, López-Ferrari AR, Ramírez-Morillo I. 2005. Bromeliaceae. Flora de Veracruz 136: 1307.

Espejo-Serna A, López-Ferrari AR, Ramírez-Morillo I. 2010. Bromeliaceae. Flora del Bajío y Regiones Adyacentes 165: 1-82.

Espejo-Serna A, López-Ferrari AR, Ramírez-Morillo I, Holst BK, Luther HE, Till W. 2004. Checklist of Mexican Bromeliaceae with notes on species distribution and levels of endemism. Selbyana 25: 33-86.

Espejo-Serna A, López-Ferrari AR, Ramírez-Morillo I, Martínez-Correa N. 2007a. Dos nuevas especies de Hechtia (Bromeliaceae) de México. Acta Botanica Mexicana 78: 97-109. DOI: 10.21829/ abm78.2007.1033

Espejo Serna A, López-Ferrari AR, Till W. 2007b. Tillandsia suesilliae Espejo, López-Ferrari et W. Till, A New Species From Central Mexico. Acta Botanica Mexicana 78: 85-95 DOI: 10.21829/ abm78.2007.1032

ESRI. 2009. Arc View GIS 9.3.1. Redlands, California.

Flores-Argüelles A, Espejo-Serna A, López-Ferrari AR. 2017. Pitcairnia singularis (Pitcairnioideae, Bromeliaceae), a new species from Jalisco, Mexico. Phytotaxa 291: 275-280. DOI: 10.11646/phytotaxa.291.4.4

Flores-Cruz M, Diego-Escobar MV. 2008. Una nueva especie de Tillandsia (Bromeliaceae) de Guerrero, México. Boletín de la Sociedad Botánica de México 82: 15-20. DOI: 10.17129/botsci.1778.

Flores-Palacios A, García-Franco JG, Valencia-Diaz S, Solis-Montero L, Cruz-Angón A. 2011. Diversidad y conservación de plantas epífitas vasculares en el centro del estado. In: CONABIO [Comisión Nacional para el Conocimiento y Uso de la Biodiversidad]. La biodiversidad en Veracruz: Estudio de Estado. México: CONABIO, Gobierno del Estado de Veracruz, Universidad Veracruzana, Instituto de Ecología A.C. 493-501. ISBN: 978-607-7607-50-2 
Flores-Palacios A, Valencia-Diaz S. 2007. Local ilegal trade reveals unknown diversity and involves a high species richness of wild vascular epiphytes. Biological Conservation 136: 372-378. DOI: 10.1016/ j.biocon.2006.12.017

García-Franco JG, Castillo-Campos G, Melthreter K, Martínez ML, Vázquez G. 2008. Conposición florística de un bosque mesófilo del centro de Veracruz, México. Boletín de la Sociedad Botánica de México 83: 37-52. DOI: $10.17129 /$ botsci. 1787

García-Franco JG, Toledo-Aceves T. 2008. Capítulo 5. Epífitas Vasculares (Bromelias y Orquídeas). In: Manson RH, Hernández-Ortiz V, Gallina S, Melthreter K, eds. Agroecosistemas cafetaleros de Veracruz. Biodiversidad, Manejo y Conservación. México: INE-SEMARNAT. 69-82. ISBN: 970-709112-6

García-Martínez R, Beutelspacher CR. 2017. Una nueva especie de Tillandsia L. (Bromeliaceae: Tillandsioideae) de Chiapas, México. Lacandonia 11: 19-28.

García-Mendoza AJ. 2004. Integración del conocimiento florístico del estado. In: García-Mendoza AJ, Ordóñez MJ, Briones-Salas M, eds. Biodiversidad de Oaxaca. México: Universidad Nacional Autónoma de México, Fondo Oaxaqueño para la Conservación de la Naturaleza, World Wildlife Fund. 302-325. ISBN: 9703220452

García-Ruiz I, Espejo-Serna A, López-Ferrari, AR. 2014. Novedades de la familia Bromeliaceae para el noroccidente de Michoacán, México. Acta Botanica Mexicana 107: 9-18. DOI: 10.21829/ abm107.2014.211

Gardner CS. 1982. A Systematic Study of Tillandsia Subgenus Tillandsia. PhD Thesis. Graduate College of Texas A\&M University.

Gardner CS. 1986. Preliminary classification of Tillandsia based on floral characters. Selbyana 9: 130146.

Givnish TJ. 2017. One Hundred Million Years of Bromeliad Evolution. Journal of the Bromeliad Society 66: $200-225$.

Givnish TJ, Barfuss MHJ, Van Ee B, Riina R, Schulte K, Horres R, Gonsiska PA, Jabaily RS, Crayn DM, Smith JAC, Winter K, Brown GK, Evans TM, Holst BK, Luther H, Till W, Zizka G, Berry PE, Sytsma KJ. 2011. Phylogeny, adaptive radiation, and historical biogeography in Bromeliaceae: Insights from an eight-locus plastid phylogeny. American Journal of Botany 98: 872-895. DOI: 10.3732/ajb.1000059

Givnish TJ, Barfuss MHJ, Van Ee B, Riina R, Shulte K, Horres R, Gonsiska PA, Jabaily RS, Crayn DM, Smith JAC, Winter K, Brown GK, Evans TM, Holst BK, Luther H, Till W, Zizka G, Berry PE, Sytsma KJ. 2014. Adaptative radiation correlated and contingent evolution, and net-species diversification in Bromeliaceae. Molecular Phylogenetics and Evolution 71: 55-78. DOI: 10.1016/j.ympev.2013.10.010

Givnish TJ, Millam KC, Berry PE, Sytsma KJ. 2007. Phylogeny, adaptive radiation, and historical biogeography of Bromeliaceae inferred from ndhF sequence data. Aliso 23: 3-26.

Givnish TJ, Sytsma KJ, Smith JF, Hahn WS. 1992. Molecular evolution, phylogeny, and geography in the Pitcairnioideae (Bromeliaceae). American Journal Botany 79: 145.

González-Rocha E, Cerros-Tlatilpa R, Espejo-Serna A, López-Ferrari AR. 2015. Tillandsia chalcatzingensis, a new species from the state of Morelos, Mexico. Phytotaxa 227: 182-188. DOI: 10.11646/ phytotaxa.227.2.8

González-Rocha E, Espejo-Serna A, López-Ferrari AR, Cerros-Tlatilpa R. 2016. Las Bromeliaceae del Estado de Morelos. Ciudad de México: Universidad Autónoma Metropolitana. ISBN: 978-607-280832-4

González-Rocha E, López-Ferrari AR, Cerros-Tlatilpa R, Espejo-Serna A. 2014. Una nueva especie de Hechtia (Bromeliaceae; Hechtioideae) del estado de Morelos, México. Acta Botanica Mexicana 109: 45-54. DOI: 10.21829/abm109.2014.1147

Google. 2016. Google Earth V.7.1.2.2041. <http://earth.google.com> (Consultado 2016).

Gouda EJ, Butcher D, Gouda CS. (actualizado continuamente). Encyclopaedia of Bromeliads, version 3. Botanic Gardens, Utrecht <http://encyclopaedia.florapix.nl/.University>. (Consultado: feb, 2018).

Granados-Mendoza C, Salazar GA, Flores-Cruz M. 2016. A New Species of the Mexican Tillandsia erubescens Group (Bromeliaceae). Phytotaxa 260: 57-65. DOI: 10.11646/phytotaxa.260.1.6

Granados-Mendoza C, Granados-Aguilar X, Donadío S, Salazar GA, Flores-Cruz M, Hágsater E, Starr JR, Ibarra-Manríquez G, Fragoso-Martínez I, Magallón S. 2017. Geographic structure in two highly diverse lineages of Tillandsia (Bromeliaceae). Botany 95: 641-651. DOI: 10.1139/cjb-2016-0250

Hernández-Cárdenas R, González-Rocha E, Espejo-Serna A, López-Ferrari AR, Cerros-Tlatilpa R, Ehlers R. 2014. Tillandsia religiosa, a new species from the state of Morelos, Mexico. Phytotaxa 184: 53-57. DOI: $10.11646 /$ phytotaxa.184.1.7

Hornung-Leoni CT, Márquez J, Bueno-Villegas, J. 2011. Arthropods associated with Tillandsia deppeana (Bromeliaceae) from Hidalgo State, México, with three first State records of Coleoptera Species. Entomological News 122: 469-476. DOI: 10.3157/021.122.0508 
INEGI [Instituto Nacional de Estadística y Geografía]. 2016. Mapas topográficos. $<$ http://www.beta.inegi. org.mx/app/mapas/> (Consultado 2016).

Kadmon R, Farber O, Danin A. 2003. A systematic analysis of factors affecting the performance of climatic envelope models. Ecological Applications 13: 853-867. DOI: 10.1890/1051-0761(2003)013[0853: ASAOFA]2.0.CO;2

Kearney M. 2006. Habitat, environment y niche: what are we modelling? Oikos 115: 186-191. DOI: 10.1111/j.2006.0030-1299.14908.x

Krömer T, Espejo A, López-Ferrari AR, Acebey A. 2007. Werauhia noctiflorens (Bromeliaceae), una Nueva Especie del Sureste de México y Belice. Novon 17: 336-340. DOI: 10.3417/1055-3177(2007)17[336: WNBUNE]2.0.CO;2

Krömer T, Kessler M, Lohaus G, Schmidt-Lebuhn AN. 2008. Nectar sugar composition and concentration in relation to pollination syndromes in Bromeliaceae. Plant Biology 10: 502-511. DOI: 10.1111/j.14388677.2008.00058.x

López-Ferrari AR, Espejo-Serna A. 2009. Nuevas combinaciones en monocotiledóneas mexicanas IV (Bromeliaceae, Orchidaceae). Acta Botanica Mexicana 89: 43-46. DOI: 10.21829/abm89.2009.304

López-Ferrari AR, Espejo-Serna A. 2013. Hechtia mapimiana (Bromeliaceae; Hechtioideae), una nueva especie del estado de Durango, México. Acta Botanica Mexicana 102: 89-97. DOI: 10.21829/ abm102.2013.230

López-Ferrari AR, Espejo-Serna A. 2014a. Bromeliaceae. Flora del Valle de Tehuacán-Cuicatlán 122: 1-180.

López-Ferrari AR, Espejo-Serna A. 2014b. Hechtia rubicunda (Bromeliaceae; Hechtioideae), una nueva especie de Oaxaca, México. Acta Botanica Mexicana 107: 153-164. DOI: 10.21829/abm107.2014.205

López-Ferrari AR, Espejo-Serna A, Blanco-Fernández-de-Caleya P. 2006. Circunscripción de Tillandsia chaetophylla Mez y descripción de Tillandsia sessemocinoi (Bromeliaceae: Tillandsioideae). Acta Botanica Mexicana 76: 77-88. DOI: 10.21829/abm76.2006.1020

López-Ferrari AR, Espejo-Serna A, Ceja-Romero J, Mendoza-Ruiz A. 2008. Hechtia matudae, a spectacular, though neglected Bromeliad from Mexico. Journal of Bromeliad Society 58: 49-96.

López-Ferrari AR, Espejo-Serna A, Ceja-Romero J, Mendoza-Ruiz A. 2011. Aechmea aenigmatica (Bromeliaceae; Bromelioideae) una nueva especie del estado de Oaxaca, México. Acta Botanica Mexicana 95: 1-9. DOI: $10.21829 / \mathrm{abm} 95.2011 .262$

López-Ferrari AR, Espejo-Serna MA, Martínez-Correa N. 2009. Hechtia caulescens (Bromeliaceae), a new species from central Mexico. Novon 19: 197-200. DOI: 10.3417/2007078

Martínez-Correa N, Espejo-Serna A, López-Ferrari, AR. 2014. Una nueva especie de Catopsis (Bromeliaceae, Tillandsioideae, Catopsideae) de México. Acta Botanica Mexicana 106: 129-147. DOI: 10.21829/abm106.2014.221

Martínez-Correa N, Espejo-Serna MA, López-Ferrari AR, Ramírez-Morillo I. 2010. Two Novelties in Hechtia (Bromeliaceae, Hechtioideae) from Mexico. Systematic Botany 35: 745-754. DOI: $10.1600 / 036364410 X 539835$

Matuda E. 1952. Las Bromeliáceas de Chiapas. Anales del Instituto de Biología de la Universidad Nacional de México 23: 85-153.

Matuda E. 1957. Vriesia ovandensis Matuda, sp. nov. Cactáceas y Suculentas Mexicanas 2: 78-79.

Matuda E. 1973. New additions to Tillandsia (Bromeliaceae) from Mexico. Cactus \& Succulent Journal 45: $186-189$.

Matuda E. 1975. Dos nuevas Bromeliáceas de México. Cactáceas y Suculentas Mexicanas 20: 44-46.

Matuda E. 1977. Nuevas Bromeliáceas Mexicanas I. Cactáceas y Suculentas Mexicanas 22: 20-24.

McVaugh R. 1989. Bromeliaceae to Dioscoreaceae. In: Anderson W, ed. Flora Novo-Galiciana: A descriptive account of the vascular plants of western Mexico. 15. Ann Arbor: The University of Michigan, 4-79. ISBN: 9780962073304

Mez C. 1897. Bromeliaceae. In: de Candolle C. ed. Monographie Phanerogamarum 9. Paris: Masson \& Cie. ISBN: 9788121105699

Mez C. 1935. Bromeliaceae. In: Engler A, ed. Das Pflanzenreich IV. 32: 1-667.

Mondragón-Chaparro DM, Ticktin T. 2011. Demographic effects of harvesting epiphytic bromeliads and an alternative approach to collections. Conservation Biology 25: 797-807. DOI: 10.1111/j.15231739.2011.01691.x

Mondragón D, Villa-Guzmán DM. 2008. Estudio etnobotánico de las bromelias epífitas en la comunidad de Sta. Catarina Ixtepeji. Polibotánica 26: 175-191.

Pinzón JP, Ramírez IM, Carnevali G. 2012. The re-establishment of Tillandsia cucaensis (Bromeliaceae) a good species formerly confused with a new species from the Gulf of Honduras. Phytotaxa 61: 1-16. DOI: $10.11646 /$ phytotaxa.61.1.1

Pinzón JP, Ramírez-Morillo IM, Carnevali-Fernández-Concha G. 2011. Morphometric analyses within 
Tillandsia utriculata L. complex (Bromeliaceae) allow for the recognition of a new species, with notes on its phylogenetic position. Journal of the Torrey Botanical Society 138: 353-365. DOI: 10.3159/TORREY-D-11-00005.1

Pinzón JP, Ramírez-Morillo IM, Carnevali G, Barfuss MHJ, Till W, Tun J, Ortíz-Dáz JJ. 2016. Phylogenetics and evolution of the Tillandsia utriculata complex (Bromeliaceae, Tillandsioideae) inferred from three plastid DNA markers and the ETS of the nuclear ribosomal DNA. Botanical Journal of the Linnean Society 181: 362-390. DOI: 10.1111/boj.12425

Pulido-Esparza VA, López-Ferrari AR, Espejo-Serna A. 2004. Flora bromeliológica del Estado de Guerrero, México: riqueza y distribución. Boletín de la Sociedad Botánica de México 75: 55-104. DOI: 10.17129/botsci.1693

Ramírez-Morillo I, Carnevali-Fernández-Concha G, Chi-May F. 2004. Guía ilustrada de las Bromeliaceae de la porción mexicana de la Península de Yucatán. Centro de Investigación Científica de Yucatán. ISBN: 9686532145.

Ramírez MIM, Carnevali FCG. 2003. A new speccies of Tillandsia (Bromeliaceae) from the Mexican Yucatán Peninsula. Novon 13: 209-211. DOI: 10.2307/3393522

Ramírez MIM, Carnevali FCG. 2007a. Two new species in the Tillandsia utriculata Complex (Bromeliaceae) from Mexico. Novon 17: 72-78. DOI: 10.3417/1055-3177(2007)17[72:TNSITT]2.0.CO;2

Ramírez MIM, Carnevali FCG. 2007b. A new species in the Tillandsia utriculata Complex (Bromeliaceae) from Mexico. Novon 17: 383-385. DOI: 10.3417/1055-3177(2007)17[383:ANSITT]2.0.CO;2

Ramírez-Morillo IM. 2008. A new Hechtia (Bromeliaceae) from the states of Querétaro and Hidalgo, Mexico. Acta Botanica Mexicana 85: 63-74. DOI: 10.21829/abm85.2008.1073

Ramírez-Morillo IM, Espejo-Serna A, López-Ferrari AR. 2011. A new species of Hechtia (Bromeliaceae) from Chihuaha, Mexico. Novon 21: 362-367. DOI: 10.3417/2009130

Ramírez-Morillo IM, Jiménez-Nah CF. 2012. Hechtia aquamarina, a new name for Hechtia pueblensis I. Ramírez \& Jiménez. Phytotaxa 48: 33. DOI: 10.11646/phytotaxa.48.1.5

Ramírez-Morillo I, Jiménez-Nah CF, Treviño-Carreón J. 2013. A new species of Hechtia (Bromeliaceae) from southwestern Tamaulipas, Mexico. Phytotaxa 112: 33-42. DOI: 10.11646/phytotaxa.112.2.1

Ramírez-Morillo I, Hornung-Leoni CT, González-Ledesma M, Treviño-Carreón J. 2015. A new species of Hechtia (Bromeliaceae: Hechtioideae) from Hidalgo (Mexico). Phytotaxa 221: 157-165. DOI: 10.11646/phytotaxa.221.2.5

Ramírez-Morillo I, Jiménez CF, Carnevali-Fernández-Concha G, Pinzón JP. 2014. Three new species and growth patterns in Hechtia (Bromeliaceae: Hechtioideae). Phytotaxa 178: 113-127. DOI: 10.11646/ phytotaxa.178.2.3

Rzedowski J. 1991. El endemismo en la flora fanerogámica mexicana: una apreciación analítica preliminar. Acta Botanica Mexicana 15: 47-64. DOI: 10.21829/abm15.1991.620

Salazar-Rodríguez JL. 2010. Holoepifitas Vasculares del Bosque Mesófilo de Montaña del Municipio de Acajete, Veracruz. Bsc. Thesis. Universidad Veracruzana.

Sarukhán J. 2008. Presentación In: Soberón J, Halfter G, Llorente-Bousquets J, comps. Capital Natural de México. Volumen I Conocimiento Actual de la Biodiversidad. México: CONABIO. ISBN- 978-6077607-03-8

Schütz N, Krapp F, Wagner N, Weising K. 2016. Phylogenetics of Pitcairnioideae s.s. (Bromeliaceae): evidence from nuclear and plastid DNA sequence data. Botanical Journal of the Linnean Society 181: 323-342. DOI: 10.1111/boj.12403

Sessé M, Mociño JM. 1893. Plantae Novae Hispaniae. Editio secunda. México: Oficina tipográfica de la Secretaria de Fomento.

Sessé M, Mociño JM. 1894 Flora Mexicana. Editio secunda. México: Oficina tipográfica de la Secretaría de Fomento.

Smith LB. 1938. Parte 2. (Xiridiales) Bromeliaceae. In: North American Flora. New York Botanical Garden. 19: 61-228.

Smith LB, Downs RJ. 1974. Pitcairnioideae (Bromeliaceae). Flora Neotropica 14: 1-658. ISBN: 978-089327-303-3

Smith LB, Downs RJ. 1977. Tillandsioideae (Bromeliaceae). Flora Neotropica 14: 659-1492. ISBN: 9780-89327-334-7

Smith LB, Downs RJ. 1979. Bromelioideae (Bromeliaceae). Flora Neotropica 14: 1493-2142. ISBN: 978 0-89327-210-4

Thiers B. [continuously updated]. Index Herbariorum: A global directory of public herbaria and associated staff. New Yorg Botanical Garden's Virtual Herbarium. <http://sweetgum.nybg.org/science/ih $>$ (Consultado: novemeber 2107)

Till W. 1992. A well-known new species: Tillandsia fuchsii. Journal of the Bromeliad Society 42: 99 102. 
Till W, Schatzl S. 1981. Eine neue Tillandsia-Art aus Südmexico: T. kolbii (Bromeliaceae). Plants Systematics and Evolution 138: 259-262. DOI: 10.1007/BF00985189

Till W, Halbritter H, Zecher E. 1989. A remarkable, New, Large Tillandsia from Western Mexico. Journal of the Bromeliad Society 39: 152-156.

Utley JF, Burt-Utley K. 1994. Bromeliaceae In: Davidse G, Sousa-Sánchez M, Chater AO. eds. Flora Mesoamericana. México: Universidad Nacional Autónoma de México, The Natural History Museum and Missouri Botanical Garden. 89-156.

Viccon-Esquivel J. 2009. Riqueza y Composición Florística de las Epífitas Vasculares del Bosque Mesófilo de Montaña de las Localidades de Atzalan y Zongolica, Veracruz. BSc. Thesis. Universidad Veracruzana.

Victoria-Hernández A. 2001. Bromeliaceae In: Calderón de Rzedowski G, Rzedowski J eds. Flora Fanerogámica del Valle de México. Pátzcuaro, Michoacán: Instituto de Ecología A.C.- CONABIO. 11791187. ISBN 970-9000-17-9

Villa-Guzmán DM. 2007. Estudio Etnobotánico de las Bromelias Epifitas de Santa Catarina Ixtepeji, en la Sierra Norte de Oaxaca. MSc. Thesis. CIIDIR Oaxaca: Instituto Politécnico Nacional.

Villaseñor JL. 2016. Checklist of the native vascular plants of Mexico. Revista Mexicana de Biodiversidad 87: 559-902. DOI: 10.1016/j.rmb.2016.06.017

Wolf JHD, Konings CJF. 2001. Towards the sustainable harvesting of epiphytic bromeliads: a pilot study from the highlands of Chiapas, Mexico. Biological Conservation 101: 23-31. DOI: 10.1016/S00063207(01)00053-2 
Apéndice 1. Listado de las especies de Bromeliaceae presentes en México; después del nombre del género se indica, entre paréntesis, el número total de especies que éste incluye, el número de especies presentes en el territorio nacional, el número de especies endémicas de México y el número de las que restringen su distribución a una sola entidad (microendémicas), las cuales se señalan en el listado con un asterisco ${ }^{*}$ ).

Aguascalientes (AGS), Baja California (BC), Baja California Sur (BCS), Campeche(CAM), Ciudad de México $(\mathrm{CDMX})$, Chiapas (CHIS), Chihuahua $(\mathrm{CHIH})$, Coahuila $(\mathrm{COAH})$, Colima $(\mathrm{COL})$, Durango (DGO), Estado de México (MÉX), Guanajuato (GTO), Guerrero (GRO), Hidalgo (HGO), Jalisco (JAL), Michoacán (MICH), Morelos (MOR), Nayarit (NAY), Nuevo León (NL), Oaxaca (OAX), Puebla (PUE), Querétaro (QRO), Quintana Roo (QROO), San Luis Potosí (SLP), Sinaloa (SIN), Sonora (SON), Tabasco (TAB), Tamaulipas (TAM), Tlaxcala (TLX), Veracruz (VER), Yucatán (YUC), Zacatecas (ZAC).

Aechmea (248/9/2/1)

* Aechmea aenigmatica López-Ferr., Espejo, Ceja \& A. Mend. (OAX)

Aechmea bracteata (Sw.) Griseb. (CAM, CHIS, COL, GRO, HGO, JAL, MICH, NAY OAX, PUE QRO, QROO, SLP, SIN, TAB, TAM, VER, YUC)

Aechmea bromeliifolia (Rudge) Baker (CAM, QROO)

Aechmea lueddemanniana (K. Koch) Mez (CHIS, OAX, VER)

Aechmea magdalenae (André) André ex Baker (CHIS, OAX, QROO, VER)

Aechmea matudae L.B. Sm.(CHIS)

Aechmea mexicana Baker (CHIS, GRO, OAX, PUE, SLP, VER)

Aechmea nudicaulis (L.) Griseb. (CHIS, OAX, PUE, VER)

Aechmea tillandsioides (Mart. ex Schult. \& Schult. f.) Baker (CAM, CHIS, OAX, QROO, TAB, VER)

Androlepis (2/1/0/0)

Androlepis skinneri (K. Koch) Brongn. ex Houllet(CHIS, TAB, VER)

Billbergia (64/2/0/0)

Billbergia pallidiflora Liebm. (CHIS, COL, GRO, JAL, MICH, NAY, OAX, SIN)

Billbergia viridiflora $\mathrm{H}$. Wendl. (CHIS, TAB)

Bromelia (71/4/2/0)

Bromelia hemisphaerica Lam. (MÉX, MOR, OAX)

Bromelia karatas L. (CAM, CHIS, GRO, JAL, MICH, NAY OAX, QRO, SIN, SLP, VER, YUC)

Bromelia palmeri Mez (COL, GRO, JAL, MICH, NAY, OAX)

Bromelia pinguin L. (CHIS, COL, GRO, JAL, MICH, NAY, OAX, QRO, QROO, SIN, SLP, SON, TAB, TAM,

VER, YUC)

Catopsis $(18 / 17 / 6 / 1)$

Catopsis berteroniana (Schult. \& Schult. f.) Mez (CAM, CHIS, QROO, TAB)

* Catopsis compacta Mez (OAX)

Catopsis delicatula L.B. Sm. (CHIS, OAX, VER)

Catopsis floribunda L.B. Sm. (CHIS, OAX)

Catopsis juncifolia Mez \& Wercklé ex Mez (CHIS, VER)

Catopsis minimiflora Matuda (CHIS)

Catopsis morreniana Mez (CHIS, OAX, PUE, QROO, TAB, VER)

Catopsis nitida (Hook.) Griseb. (CHIS, OAX, VER)

Catopsis nutans (Sw.) Griseb. (CAM, CHIS, COL, GRO, JAL, MEX, MICH, MOR, NAY, OAX, PUE, QROO,

TAB, TAMP, VER)

Catopsis occulta Mart.-Correa, Espejo \& López-Ferr. (CHIS, OAX, VER)

Catopsis oerstediana Mez (CHIS)

Catopsis paniculata E. Morren (OAX, PUE, VER)

Catopsis pendula Baker (GRO, JAL, MEX, MICH, MOR, NAY)

Catopsis sessiliflora (Ruiz \& Pav.) Mez (CAM, CHIS, GRO, HGO, JAL, MICH, OAX, PUE, VER)

Catopsis subulata L.B. Sm. (CHIS, OAX, PUE, VER)

Catopsis wangerinii Mez \& Wercklé ex Mez (CHIS, VER)

Catopsis wawranea Mez (CHIS, OAX, VER)

Fosterella (31/1/0/0)

Fosterella micrantha (Lindl.) L.B. Sm. (CHIS, GRO, JAL, MICH, OAX, VER)

Greigia (35/3/3/0)

Greigia juareziana L.B. Sm. (CHIS, OAX)

Greigia oaxacana L.B. Sm. (OAX, VER)

Greigia van-hyningii L.B. Sm. (CHIS, OAX, VER)

Guzmania (219/2/0/0)

Guzmania lingulata (L.) Mez (CHIS, OAX)

Guzmania nicaraguensis Mez \& C. F. Baker ex Mez (CHIS, OAX, VER)

Hechtia (76/71/70/46)

Hechtia aquamarina I. Ramírez \& C. F. Jiménez (OAX, PUE)

* Hechtia argentea Baker (HGO) 
Hechtia bracteata Mez (PUE, VER)

* Hechtia carlsoniae Burt-Utley \& Utley (GRO)

* Hechtia caudata L.B. Sm. (OAX)

Hechtia caulescens López-Ferr., Espejo \& Mart.-Correa (MOR, OAX)

* Hechtia chichinautzensis Mart.-Correa, Espejo \& López-Ferr. (MOR)

* Hechtia colossa Mart.-Correa, Espejo \& López-Ferr. (OAX)

* Hechtia complanata Burt-Utley (OAX)

Hechtia confusa L.B. Sm. (OAX, PUE)

Hechtia conzattiana L.B. Sm. (OAX, PUE)

* Hechtia deceptrix I. Ramírez \& C.T. Hornung (HGO)

* Hechtia edulis I. Ramírez, Espejo \& López-Ferr. (CHIH)

* Hechtia elliptica L.B. Sm. (COAH)

* Hechtia epigyna Harms (TAM)

* Hechtia flexilifolial. Ramírez \& Carnevali (OAX)

* Hechtia fosteriana L.B. Sm. (OAX)

Hechtia fragilis Burt-Utley \& Utley (OAX, PUE)

* Hechtia galeottii Mez (OAX)

* Hechtia ghiesbreghtii Lem. (CHIS)

* Hechtia glabra Brandegee (VER)

* Hechtia glauca Burt-Utley \& Utley (MICH)

Hechtia glomerata Zucc. (AGS, GTO, HGO, MEX, NL, QRO, SLP, TAM)

* Hechtia hernandez-sandovalii I. Ramírez, C. F. Jiménez \& Treviño (TAM)

Hechtia hintoniana Burt-Utley, Utley \& García-Mendoza (MEX, MICH)

* Hechtia huamelulaensis I. Ramírez \& Carnevali (OAX)

* Hechtia iltisii Burt-Utley \& Utley (JAL)

* Hechtia isthmusiana Burt-Utley (OAX)

* Hechtia ixtlanensis Burt-Utley (OAX)

* Hechtia jaliscana L.B. Sm. (JAL)

* Hechtia laevis L.B. Sm. (COL)

* Hechtia lanata L.B. Sm. (OAX)

* Hechtia laxissima L.B. Sm. (MICH)

Hechtia liebmannii Mez (PUE, VER)

Hechtia lundelliorum L.B. Sm. (HGO, PUE, QRO, SLP)

Hechtia lyman-smithii Burt-Utley \& Utley (OAX, PUE)

* Hechtia mapimiana López-Ferr. \& Espejo (DGO)

* Hechtia marnier-lapostollei L.B. Sm. (OAX)

Hechtia matudae L.B. Sm. (MÉX, MOR)

* Hechtia melanocarpa L.B. Sm. (GRO)

Hechtia mexicana L.B. Sm. (SLP, TAM)

Hechtia montana Brandegee (BCS, SON)

* Hechtia montis-frigidi Gonz.-Rocha, Espejo, López-Ferr. \& Cerros (MOR)

* Hechtia mooreana L.B. Sm. (GRO)

* Hechtia myriantha Mez (VER)

* Hechtia nivea I. Ramírez \& C. F. Jiménez (OAX)

* Hechtia nuusaviorum Espejo \& López-Ferr. (OAX)

* Hechtia oaxacana Burt-Utley, Utley \& García-Mend. (OAX)

* Hechtia pedicellata S. Watson (JAL)

Hechtia podantha Mez (AGS, GTO, HGO, QRO)

* Hechtia pretiosa Espejo \& López-Ferr. (GTO)

* Hechtia pringlei B.L. Rob. \& Greenm. (OAX)

* Hechtia pueblensis Burt-Utley, Utley \& García-Mend. (PUE)

* Hechtia pumila Burt-Utley \& Utley (GRO)

* Hechtia purhepecha I. García, Espejo \& López-Ferr. (MICH)

* Hechtia purpusii Brandegee (VER)

Hechtia reflexa L.B. Sm. (GRO, MICH)

Hechtia reticulata L.B. Sm. (COL, JAL, MICH)

Hechtia rosea É. Morren ex Baker (CHIS, OAX)

Hechtia roseana L.B. Sm. (OAX, PUE)

* Hechtia rubicunda López-Ferr. \& Espejo (OAX)

* Hechtia santanae I. Ramírez \& P. Carrillo (JAL)

Hechtia schottii Baker (CAM, YUC)

Hechtia sphaeroblasta B.L. Rob. (GRO, OAX, PUE)

* Hechtia stenopetala Klotzsch (VER)

* Hechtia suaveolens É. Morren ex Mez (OAX)

Hechtia subalata L.B. Sm. (DGO, JAL, NAY, ZAC) 
Hechtia tehuacana B.L. Rob. (OAX, PUE)

Hechtia texensis S. Watson(COAH, DGO, NL, ZAC)

Hechtia tillandsioides (André) L.B. Sm. (GRO, MÉX, QRO, SLP)

* Hechtia zamudioi Espejo, López-Ferr. \& I. Ramírez (QRO)

Hohenbergiopsis (1/1/0/0)

Hohenbergiopsis guatemalensis (L.B. Sm.) L.B. Sm. \& Read OAX

Pitcairnia (406/50/40/20)

Pitcairnia abundans L.B. Sm. (NAY, OAX)

Pitcairnia amblyosperma L.B. Sm. (PUE, SLP, VER)

Pitcairnia breedlovei L.B. Sm. (CHIS, OAX)

Pitcairnia calderonii Standl. \& L.B. Sm. (CHIS)

Pitcairnia carioana Wittm. (CHIS)

* Pitcairnia chiapensis Miranda (CHIS)

Pitcairnia colimensis L.B. Sm. (COL, MICH)

Pitcairnia compostelae McVaugh (JAL, NAY)

Pitcairnia cylindrostachya L.B. Sm. (JAL, NAY)

* Pitcairnia densiflora Brongn. ex Lem. (VER)

* Pitcairnia flavescentia Matuda (GRO)

Pitcairnia flexuosa L.B. Sm. (MEX, MICH)

Pitcairnia foliacea L.B. Sm. (GRO, JAL, MICH)

Pitcairnia heterophylla (Lindl.) Beer (CHIS, COL, GRO, JAL, MEX, MOR, NAY, OAX, SIN, VER)

Pitcairnia hintoniana L.B. Sm. (GRO, MEX)

Pitcairnia imbricata (Brongn.) Regel (CHIS, GRO, JAL, NAY, OAX, PUE, VER)

Pitcairnia jaliscana S. Watson (GTO, JAL, MICH, ZAC)

Pitcairnia karwinskyana Schult. \& Schult. f. (GRO, MEX)

* Pitcairnia leprosa L.B. Sm. (GRO)

* Pitcairnia matudae L.B. Sm. (CHIS)

Pitcairnia micheliana André (COL, JAL, MICH)

* Pitcairnia micropoda L.B. Sm. (MEX)

* Pitcairnia mirandae Utley \& Burt-Utley (CHIS)

* Pitcairnia modesta L.B. Sm. (GRO)

* Pitcairnia monticola Brandegee (SIN)

* Pitcairnia mooreana L.B. Sm. (GRO)

Pitcairnia oaxacana L.B. Sm. (GRO, NAY, OAX)

* Pitcairnia ocotensis Beutelsp. \& G. López V. (CHIS)

Pitcairnia palmeri S. Watson var. palmeri(CHIH, JAL, MOR, NAY, SIN, ZAC)

Pitcairnia palmeri S. Watson var. longebracteataL.B. Sm. (DGO, SIN)

Pitcairnia pteropoda L.B. Sm. (GRO, MICH)

Pitcairnia punicea Scheidw. (CHIS, TAB, VER)

* Pitcairnia queroana Espejo \& López-Ferr. (CHIS)

Pitcairnia recurvata (Scheidw.) K. Koch (CHIS, OAX, TAB, VER)

Pitcairnia ringens Klotzsch (HGO, MEX, OAX, PUE, QRO, SLP, TAM, VER)

Pitcairnia roseana L.B. Sm. (DGO, NAY)

Pitcairnia saxicola L.B. Sm. (CHIS)

Pitcairnia schiedeana Baker (OAX, VER)

* Pitcairnia secundiflora L.B. Sm. (CHIS)

* Pitcairnia singularis Flores-Arg., Espejo \& López-Ferr. (JAL)

* Pitcairnia sordida L.B. Sm. (GRO)

* Pitcairnia tabuliformis Linden (CHIS)

* Pitcairnia tillandsioides L.B. Sm.(GRO)

Pitcairnia tuerckheimii Donn. Sm. (CHIS, OAX)

Pitcairnia undulata (hort. ex Beer) Schiedw. (CHIS, OAX, TAB, VER)

* Pitcairnia vallisoletana Lex. (MICH)

* Pitcairnia virginalis Utley \& Burt-Utley (OAX)

Pitcairnia wendlandii Baker (CHIS, GRO, OAX)

Pitcairnia xanthocalyx Mart. (QRO, SLP)

* Pitcairnia yocupitziae Espejo \& López-Ferr. (GRO)

Pseudalcantarea (3/3/0/0)

Pseudalcantarea grandis (Schltdl.) Pinzón \& Barfuss (GTO, OAX, PUE, QRO, VER)

Pseudalcantarea macropetala (Wawra) Pinzón \& Barfuss (CHIS, OAX, PUE, VER)

Pseudalcantarea viridiflora (Beer) Pinzón \& Barfuss (CHIS, HGO, OAX, PUE, QRO, SLP, TAM, VER)

Racinaea $(78 / 2 / 0 / 0)$

Racinaea ghiesbreghtii (Baker) M.A. Spencer \& L.B. Sm. (OAX, VER)

Racinaea rothschuhiana (Mez) M.A. Spencer \& L.B. Sm. (CHIS, OAX, VER)

Tillandsia (705/230[8]/177/99) 
Tillandsia achyrostachys E. Morren ex Baker var. achyrostachys (COL,GRO, GTO, JAL, MEX, MICH, MOR, NAY, OAX, PUE, SIN, SON, ZAC)

* Tillandsia achyrostachys E. Morren ex Baker var. stenolepis L.B. Sm. (MICH)

* Tillandsia aguascalientensis C. S. Gardner (AGS)

Tillandsia albida Mez \& Purpus (GTO, HGO, QRO)

* Tillandsia x alfredo-lauii Rauh \& J. Lehm. (HGO)

* Tillandsia alvareziae Rauh (VER)

Tillandsia andrieuxii (Mez) L.B. Sm. (CDMX, GRO, HGO, MEX, MICH, MOR)

Tillandsia arroyoensis (W. Weber \& Ehlers) Espejo \& López-Ferr. (CHIH, COAH, NL, SLP, TAM)

* Tillandsia atenangoensis Ehlers \& Wülfingh. (OAX)

* Tillandsia atrococcinea Matuda (OAX)

* Tillandsia atroviolacea Ehlers \& Koide (OAX)

Tillandsia baileyi Rose ex Small (TAM)

Tillandsia balbisiana Schult. \& Schult. f. (CAM, CHIS, COL, GRO, JAL, NAY, OAX, QROO, SLP, SIN, TAB, VER, YUC)

Tillandsia bartramii Elliott (GTO, HGO, NL, QRO, SLP, TAM, VER)

Tillandsia belloensis W. Weber (CHIS, OAX, VER)

Tillandsia x bergianaTakiz. \& Koide (CHIS)

* Tillandsia beutelspacheri Matuda (CHIS)

* Tillandsia bochilensis Ehlers (CHIS)

Tillandsia borealis López-Ferr. \& Espejo (DGO, NAY, SIN)

* Tillandsia botterii É. Morren ex Baker (VER)

Tillandsia bourgaei Baker (GRO, GTO, JAL, MEX, MICH, MOR, NAY, OAX, PUE)

Tillandsia brachycaulos Schltdl. (CAM, CHIS, OAX, QRO, QROO, TAB, VER, YUC)

Tillandsia bulbosa Hook. (CAM, CHIS, QROO, TAB, VER)

Tillandsia butzii Mez (CHIS, GRO, JAL, OAX, PUE, VER)

Tillandsia califani Rauh (OAX, PUE)

* Tillandsia callichroma L. Hrom. (OAX)

Tillandsia calothyrsus Mez (COL, GRO, JAL, NAY, OAX)

* Tillandsia capistranoensis Ehlers \& W. Weber (ZAC)

Tillandsia capitata Griseb. (CHIS, GRO, JAL, MEX, MICH, MOR, NAY, OAX, SIN, SON)

Tillandsia caput-medusae É. Morren (CHIS, CHIH, COL, DGO, GRO, JAL, MEX, MICH, MOR, NAY, OAX, SIN, SON, ZAC)

* Tillandsia carlos-hankii Matuda (OAX)

* Tillandsia carlsoniae L.B. Sm. (CHIS)

* Tillandsia celata Ehlers \& Lautner (OAX)

Tillandsia chaetophylla Mez (OAX, VER)

* Tillandsia chalcatzingensis Gonz.-Rocha, Cerros, López-Ferr. \& Espejo (MOR)

* Tillandsia chapalillaensis Ehlers \& Lautner (NAY)

* Tillandsia chiapensis C.S. Gardner (CHIS)

Tillandsia chlorophylla L.B. Sm. (CHIS, OAX, VER)

Tillandsia circinnatioides Matuda (GRO, MOR, OAX, PUE)

* Tillandsia coalcomanensis Ehlers (MICH)

* Tillandsia comitanensis Ehlers (CHIS)

Tillandsia compressa Bertero ex Schult. \& Schult. f. (CHIS, OAX, VER)

Tillandsia concolor L.B. Sm. (GRO, OAX, VER)

* Tillandsia copalaensis Ehlers (OAX)

Tillandsia cossonii Baker (CDMX, GTO, GRO, JAL, MICH, MOR)

Tillandsia cretacea L.B. Sm. (CHIH, SON)

* Tillandsia crista-galli Ehlers (CHIS)

Tillandsia cryptantha Baker (MEX, MOR)

Tillandsia cucaensis Wittm. (CHIS, GRO, JAL, MICH, OAX)

* Tillandsia x cuchnichim R. Guess \& V. Guess (CHIS)

Tillandsia dasyliriifolia Baker (CAM, CHIS, QROO, TAB, YUC)

Tillandsia deflexa L.B. Sm. (CHIS)

* Tillandsia delicata Ehlers (OAX)

Tillandsia deppeana Steud. (GTO, HGO, PUE, QRO, SLP, TAM, VER)

Tillandsia diguetii Mez \& Rol.-Goss. (COL, JAL)

Tillandsia dugesii Baker (AGS, GTO, JAL, MEX, MICH, OAX)

* Tillandsia durangensis Rauh \& Ehlers (DGO)

* Tillandsia ehlersiana Rauh (CHIS)

Tillandsia eistetteri Ehlers (COL, GRO, JAL, MICH, NAY, OAX, SIN)

Tillandsia eizii L.B. Sm. (CHIS)

* Tillandsia elizabethae Rauh (CHIH)

Tillandsia elongata Kunth var. subimbricata (Baker) L.B. Sm. (CAM, QROO, TAB, YUC) 
* Tillandsia elusiva Pinzón, I. Ramírez \& Carnevali (CHIS)

Tillandsia erubescens Schltdl. (AGS, CDMX, CHIH, DGO, GTO, HGO, JAL, MEX, MICH, OAX, PUE, QRO,

SIN, SLP, SON, TLX, VER, ZAC)

* Tillandsia escahuascensis Espejo, López-Ferr., Ceja \& A.Mend. (PUE)

Tillandsia excelsa Griseb. (CHIS)

Tillandsia exserta Fernald (SIN, SON)

Tillandsia fasciculata Sw. (CAM, CHIS, QROO, TAB, YUC)

* Tillandsia fascifolia Flores Cruz \& Diego-Esc. (GRO)

Tillandsia ferrisiana L.B. Sm. (BCS, NAY, SIN)

Tillandsia festucoides Brongn. ex Mez (CAM, CHIS, OAX, QROO, TAB, VER)

Tillandsia filifolia Schltdl. \& Cham.(CHIS, HGO, OAX, PUE, TAB, VER)

Tillandsia flabellata Baker (CHIS, OAX)

Tillandsia flavobracteata Matuda (OAX, VER)

Tillandsia flexuosa Sw. (CAM, QROO, YUC)

* Tillandsia floresensis Ehlers (CHIS)

Tillandsia foliosa M. Martens \& Galeotti (OAX, PUE, VER)

Tillandsia fresnilloensis W. Weber \& Ehlers (DGO, JAL, ZAC)

Tillandsia fuchsii W. Till var. fuchsii (CHIS, COL, GRO, JAL, MICH, NAY, OAX)

* Tillandsia fuchsii W. Till var. stephani W. Till (JAL)

* Tillandsia glabrior (L.B. Sm.) López-Ferr., Espejo \& I. Ramírez (OAX)

* Tillandsia gracillima L.B. Sm. (PUE)

Tillandsia graebeneri Mez (SIN DATOS)

Tillandsia grossispicata Espejo, López-Ferr. \& W. Till (COL, GTO, JAL, MICH)

Tillandsia guatemalensis L.B. Sm. (CHIS, OAX)

Tillandsia guenther-nolleri Ehlers (COL JAL, MICH)

* Tillandsia guerreroensis Rauh (GRO)

Tillandsia gymnobotrya Baker (GRO, HGO, OAX, PUE, VER)

Tillandsia hammeri Rauh \& Ehlers (OAX, PUE)

Tillandsia heterophylla É. Morren (PUE, VER)

Tillandsia hintoniana L.B. Sm. (GRO, MEX, MICH, MOR)

* Tillandsia huajuapanensis Ehlers \& Lautner (OAX)

* Tillandsia huamelulaensis Ehlers (OAX)

Tillandsia hubertiana Matuda (GRO, MOR)

* Tillandsia ilseana W. Till, Halbritter \& Zecher (GRO)

Tillandsia imperialis É. Morren ex Mez (HGO, OAX, PUE, QRO, VER)

Tillandsia inopinata Espejo, López-Ferr. \& W. Till (GTO, HGO, OAX, PUE, QRO, SLP, TAM)

Tillandsia intermedia Mez (COL, GRO, JAL, MICH, NAY, SIN)

Tillandsia intumescens L.B. Sm. (MEX, MICH)

Tillandsia ionantha Planch. var. ionantha (COL, GRO, GTO, HGO, JAL, MEX, MICH, MOR, NAY, OAX, PUE, QRO, SLP, TAM, VER)

* Tillandsia ionantha Planch. var. vanhyningii M.B. Foster (CHIS)

* Tillandsia x jaguactalensis I. Ramírez, Carnevali \& Chi (QROO)

Tillandsia jaliscomonticola Matuda (COL, JAL)

* Tillandsia jaliscopinicola L. Hrom. \& P. Schneid. (JAL)

* Tillandsia juerg-rutschmannii Rauh (CHIS)

Tillandsia juncea (Ruiz \& Pav.) Poir. (CAM, CDMX, CHIS, COL, GRO, GTO, HGO, JAL, MEX, MICH, MOR,

NAY, OAX, PUE, QRO, QROO, TAB, VER, ZAC)

Tillandsia kalmbacheri Matuda (GRO, OAX)

Tillandsia karwinskyana Schult. \& Schult. f. (HGO, GTO, NL, QRO, SLP, TAM)

Tillandsia kirchhoffiana Wittm. (OAX, PUE, VER)

* Tillandsia klausii Ehlers (CHIS)

Tillandsia kolbii W. Till \& Schatzl (CHIS, OAX)

* Tillandsia lagunaensis Ehlers (OAX)

Tillandsia lampropoda L.B. Sm. var. lamproda (CHIS, OAX)

Tillandsia lampropoda L.B. Sm. var. major L.B. Sm. (CHIS)

Tillandsia langlasseana Mez (GRO, MEX, MICH, MOR)

Tillandsia laui Matuda (GRO, OAX)

Tillandsia lautneri Ehlers (CHIS, OAX)

Tillandsia leiboldiana Schltdl. (CHIS, OAX, PUE, VER)

* Tillandsia leucolepis L.B. Sm. (OAX)

Tillandsia limbata Schltdl. (CHIS, HGO, PUE, QRO, SLP, VER)

* Tillandsia loma-blancae Ehlers \& Lautner (JAL)

* Tillandsia loxichaensis Ehlers (OAX)

Tillandsia lucida E. Morren ex Baker (CHIS, OAX, VER)

* Tillandsia lydiae Ehlers (GRO) 
Tillandsia macdougallii L.B. Sm. (CDMX, DGO, GRO, GTO, HGO, JAL, MEX, MICH, NAY, OAX, PUE, TLX, VER)

Tillandsia macrochlamys Baker (OAX, PUE, VER)

Tillandsia macvaughii Espejo \& López-Ferr. (JAL, MICH)

* Tillandsia magnispica Espejo \& López-Ferr. (OAX)

Tillandsia magnusiana Wittm. (CHIS, GRO, JAL, OAX)

Tillandsia makoyana Baker (COL, DGO, GTO, JAL, MEX, MICH, MOR, NAY, OAX, PUE, SIN)

* Tillandsia makrinii L. Hrom. (OAX)

Tillandsia marabascoensis Ehlers \& Lautner (COL, GRO, JAL)

Tillandsia x marceloi Takiz. \& Koide (OAX, PUE)

Tillandsia maritima Matuda (COL, GRO, JAL, MICH, OAX)

Tillandsia mateoensis Ehlers (CHIS)

Tillandsia matudae L.B. Sm. (CHIS)

* Tillandsia maya I. Ramírez \& Carnevali (YUC)

* Tillandsia may-patii Ramírez \& Carnevali (QROO)

* Tillandsia mazatlanensis Rauh (SIN)

* Tillandsia mirabilis L. Hrom. (GRO)

* Tillandsia mitlaensis W. Weber \& Ehlers (OAX)

* Tillandsia mixtecorum Ehlers \& Koide (OAX)

Tillandsia mooreana L.B. Sm. (CHIH, DGO, JAL, NAY)

* Tillandsia moronesensis Ehlers (ZAC)

Tillandsia multicaulis Steud. (CHIS, OAX, VER)

*Tillandsia nayeliana García-Martínez \& Beutelspacher (CHIS)

Tillandsia nicolasensis Ehlers (JAL, NAY)

Tillandsia x nidus Rauh \& J. Lehm. (SIN DATOS)

Tillandsia nolleriana Ehlers (OAX, VER)

* Tillandsia novakii $\mathrm{H}$. Luther (VER)

* Tillandsia nuyooensis Ehlers (OAX)

Tillandsia oaxacana L.B. Sm. (GRO, OAX)

* Tillandsia occulta H. Luther (SIN)

Tillandsia orogenes Standl. \& L. O. Williams (CHIS)

Tillandsia ortgiesiana E. Morren ex Mez (GRO, OAX)

* Tillandsia pacifica Ehlers (JAL)

* Tillandsia pamelae Rauh (JAL)

* Tillandsia paraisoensis Ehlers (GRO)

Tillandsia parryi Baker (GTO, MEX, NL, QRO, TAM, TLX)

Tillandsia paucifolia Baker (CAM, OAX, QROO, TAM, VER, YUC)

* Tillandsia pentasticha Rauh \& Wülfingh. (GRO)

* Tillandsia pinicola I. Ramírez \& Carnevali (OAX)

Tillandsia polita L.B. Sm. var. elongata Ehlers (CHIS, OAX, VER)

Tillandsia polita L.B. Sm. var. polita (CHIS)

Tillandsia polystachia (L.) L. (CAM, CHIS, COL, GRO, HGO, JAL, NAY, OAX, PUE, QRO, TAB, TAM, VER, ZAC)

Tillandsia ponderosa L.B. Sm. (CHIS)

Tillandsia pringlei S. Watson (GTO, QRO, SLP, TAM, VER)

Tillandsia prodigiosa (Lem.) Baker (CDMX, COL, GRO, JAL, MEX, MICH, MOR, OAX, PUE)

Tillandsia pruinosa Sw. (CHIS, OAX, TAB, VER)

Tillandsia pseudobaileyi C. S. Gardner (CAM, CHIS, COL, GRO, JAL, NAY, OAX, QROO, VER)

* Tillandsia pseudooaxacana Ehlers (OAX)

Tillandsia pseudosetacea Ehlers \& Rauh (NAY, OAX)

Tillandsia pueblensis L.B. Sm. (OAX, PUE)

Tillandsia punctulata Schltdl. \& Cham. (CHIS, HGO, OAX, PUE, VER)

Tillandsia quaquaflorifera Matuda (GRO, OAX)

* Tillandsia rayonesensis Ehlers (NL)

Tillandsia x rectifolia C.A. Wiley (SIN DATOS)

Tillandsia recurvata (L.) L. (AGS, BC, BCS, CHIH, CDMX, CHIS, COAH, DGO, GRO, GTO, HGO, JAL, MEX, $\mathrm{MICH}$, MOR, NAY, NL, OAX, PUE, QRO, SIN, SLP, SON, TAM, TLX, VER, YUC, ZAC)

* Tillandsia religiosa Hern.-Cárdenas, Gonz.-Rocha, Espejo, López-Ferr., Cerros \& Ehlers (MOR)

Tillandsia remota Wittm. (CHIS)

Tillandsia rettigiana $\mathrm{Mez}(\mathrm{COL}, \mathrm{GRO}, \mathrm{JAL}, \mathrm{OAX})$

* Tillandsia rhodocephala Ehlers \& Koide (OAX)

Tillandsia rhomboidea André (CHIS)

Tillandsia rodrigueziana Mez (CHIS)

* Tillandsia roland-gosselinii Mez (COL)

* Tillandsia roseoscapa Matuda (PUE) 
* Tillandsia roseospicata Matuda (MEX)

Tillandsia rothii Rauh (COL, JAL)

Tillandsia rotundata (L.B. Sm.) C. S. Gardner (CHIS)

* Tillandsia rubrispica Ehlers \& Koide (OAX)

* Tillandsia salmonea Ehlers (CHIS)

* Tillandsia santosiae Ehlers (OAX)

* Tillandsia schatzlii Rauh (OAX)

Tillandsia schiedeana Steud. (CAM, CHIS, COL, GRO, GTO, JAL, MEX, MICH, MOR, NAY, OAX, PUE, QRO, QROO, SIN, SLP, TAB, TAM, VER, YUC)

* Tillandsia schusteri Rauh (OAX)

Tillandsia seleriana Mez (CHIS, JAL, MICH, NAY, OAX)

Tillandsia sessemocinoi López-Ferr., Espejo \& P. Blanco (JAL, MEX, MICH, MOR)

* Tillandsia setiformis Ehlers (OAX)

Tillandsia sierrahalensis Espejo \& López-Ferr. (COL, JAL)

*Tillandsia sierrajuarezensis Matuda (OAX)

Tillandsia socialis L.B. Sm. (CHIS, OAX)

Tillandsia streptophylla Scheidw. ex E. Morren (CAM, CHIS, OAX, QROO, TAB, VER, YUC)

* Tillandsia subinflata L.B. Sm. (ZAC)

Tillandsia suesilliae Espejo, López-Ferr. \& W. Till (HGO, SLP)

* Tillandsia superinsignis Matuda (MEX)

* Tillandsia supermexicana Matuda var. pendula L. Hrom. (OAX)

* Tillandsia supermexicana Matuda var. saxicola L. Hrom. (GRO)

Tillandsia supermexicana Matuda var. supermexicana (GRO, MOR, MEX, OAX)

* Tillandsia x takizawae Ehlers \& H. Luther (PUE)

* Tillandsia taxcoensis Ehlers (GRO)

Tillandsia tehuacana I. Ramírez \& Carnevali (OAX, PUE)

* Tillandsia thyrsigera E. Morren ex Baker (MEX)

* Tillandsia tillii Ehlers (JAL)

* Tillandsia tonalaensis Ehlers (OAX)

* Tillandsia trauneri L. Hrom. (GRO)

Tillandsia tricolor Schltdl. \& Cham. (CHIS, GRO, OAX, VER)

* Tillandsia ulrici Ehlers (OAX)

Tillandsia usneoides (L.) L. (AGS, CAM, CHIS, CDMX, COAH, DGO, GRO, GTO, HGO, JAL, MEX, MICH, MOR, NAY, NL, OAX, PUE, QRO, QROO, SLP, TAB, TAM, TLX, VER, YUC)

Tillandsia utriculata L. (CHIS, GTO, OAX, QRO, QROO, TAM, YUC)

Tillandsia variabilis Schltdl. (CAM, CHIS, OAX, PUE, QROO, SLP, TAB, VER)

Tillandsia velickiana L.B. Sm. (CHIS)

Tillandsia velutina Ehlers (CHIS)

* Tillandsia ventanaensis Ehlers \& Koide (DGO)

Tillandsia verapazana Ehlers (CHIS)

Tillandsia vernardoi Rauh (OAX)

Tillandsia vicentina Standl. (CHIS)

Tillandsia violacea Baker (GRO, HGO, JAL, MEX, MICH, MOR, OAX, VER)

* Tillandsia vriesioides Matuda (CHIS)

Tillandsia weberi L. Hrom. \& P. Schneid. (JAL, MICH)

* Tillandsia wuelfinghoffii Ehlers (OAX)

Tillandsia xerographica Rohweder (CHIS, GRO, OAX)

* Tillandsia yerba-santae Ehlers (OAX)

Tillandsia yunckeri L.B. Sm. (CHIS, OAX)

* Tillandsia yutaninoensis Ehlers \& Lautner (OAX)

* Tillandsia zacualpanensis Ehlers \& Wülfingh. (MEX)

* Tillandsia zaragozaensis Ehlers (NL)

* Tillandsia zoquensis Ehlers (CHIS)

* Ursulaea $(2 / 2 / 2 / 0)$

Ursulaea macvaughii (L.B. Sm.) Read \& Baensch (COL, JAL, MICH)

Ursulaea tuitensis (Magaña \& E.J. Lott) Read \& Baensch (DGO, GRO, JAL)

* Viridantha $(12 / 12 / 12 / 5)$

Viridantha atroviridipetala (Matuda) Espejo (AGS, GRO, GTO, HGO, JAL, MEX, MICH, MOR, OAX, PUE, QRO, ZAC)

* Viridantha boqueronensis (Ehlers) inéd. (OAX)

*Viridantha caballosensis (Ehlers) inéd. (GRO)

Viridantha curvifolia (Ehlers \& Rauh) López-Ferr. \& Espejo (AGS, GTO, QRO, ZAC)

Viridantha grandispica (Ehlers) inéd. (GRO, JAL, MICH, MOR, OAX, ZAC)

Viridantha ignesiae (Mez) Espejo (GRO, JAL, MEX, MICH, MOR)

Viridantha lepidosepala (L.B. Sm.) Espejo (AGS, GTO, HGO, JAL, MEX, MICH, PUE, QRO, VER, ZAC) 
* Viridantha mauryana (L.B. Sm.) Espejo (HGO)

* Viridantha penascoensis (Ehlers \& Lautner) Espejo \& López-Ferr. (OAX)

Viridantha plumosa (Baker) Espejo (GRO, MEX, OAX, PUE)

* Viridantha teloloapanensis (Ehlers \& Lautner) inéd. (GRO)

Viridantha tortilis (Klotzsch ex Baker) Espejo (DGO, GTO, HGO, JAL, QRO, SLP)

Vriesea (225/2/1/0)

Vriesea heliconioides (Kunth) Hook. ex Walp. (CAMP, CHIS, OAX, QROO, TAB, VER)

Vriesea malzinei E. Morren (CHIS, OAX, TAB, VER)

Werauhia (92/10/2/0)

Werauhia gladioliflora (H. Wendl.)J. R. Grant (CHIS, OAX, TAB, VER)

Werauhia nephrolepis (L.B. Sm. \& Pittendr.) J.R. Grant (CHIS)

Werauhia noctiflorens T. Krömer, Espejo, López-Ferr. \& Acebey (CHIS, VER)

Werauhia nocturna (Matuda) J. R. Grant (OAX)

Werauhia nutans (L.B. Sm.) J. R. Grant (CHIS, OAX, VER)

Werauhia pectinata (L.B. Sm.) J. R. Grant (CHIS, VER)

Werauhia pycnantha (L.B. Sm.) J. R. Grant (CHIS, OAX)

Werauhia vanhyningii (L.B. Sm.) J. R. Grant (OAX, PUE, VER)

Werahuia viridiflora (Regel) J.R. Grant (CHIS)

Werauhia werckleana (Mez) J. R. Grant (CHIS, OAX)

Wittmackia (44/1/1/1)

* Wittmackia mesoamericana (I. Ramírez, Carnevali \& Cetzal) Aguirre-Santoro (QROO) 